\title{
A Critical Path Generalization of the Additive Factor Method: Analysis of a Stroop Task
}

\author{
RICHARU SCHWEICKERT \\ The University of Michigan
}

\begin{abstract}
Sternberg's additive factor method was generalized to apply to tasks involving both serial and concurrent processing. The generalization is based on the critical path method of scheduling. The effects on reaction time of factors prolonging separate processes in a task are discussed; in general these effects are interactions of a simple form. Reaction times can be used to deduce, in part, the schedule of the mental processes in a task, including their order of execution. Bounds on process durations can be derived. Often there are redundant equations so the method can be easily rejected if it does not apply. A dual task experiment by Greenwald was analyzed. In the task subjects were presented with two stimuli and made a response to each under high and low compatibility conditions. Two bottlenecks in processing were located: (a) Subjects make only one decision at a time, in accordance with single channel theory, although the high compatibility condition may be an exception; (b) there is a mental process which takes longer when the stimuli conflict. The decisions about the two stimuli probably change places in the schedula when compatibility is changed.
\end{abstract}

We measure reaction times in order to make inferences about mental processes, such as perceiving, deciding, remembering, and thinking, which we cannot observe in subjects directly. 'Two methods often used to analyze reaction times, Donders' subtractive method (1968) and Sternberg's additive factor method (1969a), assume that the mental processes under consideration are performed in a sequence, one process beginning as soon as its predecessor has finished (Fig. 1). In this paper we consider more general arrangements of processes (Fig. 2).

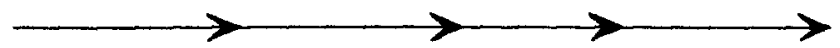

FIG. 1. A sequence of processes arranged end to end.

In both the subtractive and the additive factor methods, the amount of time required to complete a sequence of processes is considered to be the sum of the durations of all the processes in the sequence. In the subtractive method various experimental manipulations are used to insert processes into the sequence of processes or to delete them. The duration of a process can be determined by subtracting the amount of time required to complete a sequence which does not include the process from the amount of time required to complete the sequence when the process is included. 


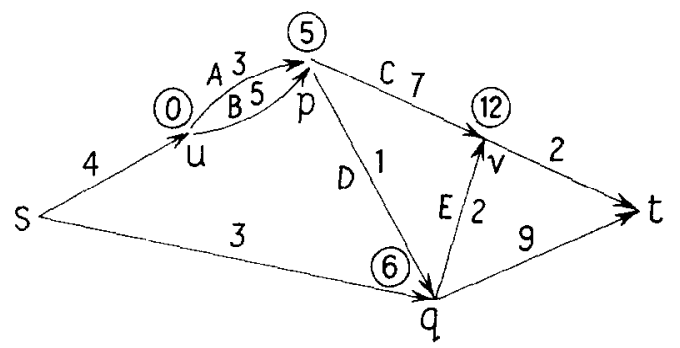

Fic. 2. A task network. Each arrow represents a process and associated with each arrow is a number giving the duration of the process. Circled numbers indicate the duration of the longest path from $u$ to the point under the circled number.

A drawback of the subtractive method is that it cannot be used to determine the order of the processes in the sequence. A more important drawback of the method is that in most cases verification of its results must rest on evidence gathered by other techniques. To use the subtractive method to make two independent measurements of the duration of some process for the purpose of verification, the process must be embedded in at least two different scquences. But it may be difficult or impossible experimentally to construct two such different sequences of processes, each complete enough psychologically to allow a subject to respond to a stimulus and yield a reaction time.

In the additive factor method the sequence always consists of the same processes, but various experimental manipulations are used to prolong the processes of interest beyond their baseline durations. For example, a visual perception process may be prolonged by making the stimulus fuzzy; a decision process may be prolonged by increasing the number of choices available to the subject. These prolongations can be made singly or in combinations. If each of several manipulations prolongs a different process, the increase in reaction time $(R T)$ produced by performing the manipulations concurrently would be the sum of the increases produced by performing them singly. Sternberg (1969a) says this additive rule is very likely to hold, although not inevitable. We see below that if all the processes are not arranged in a sequence, the effects on $R T$ of manipulations prolonging separate processes can be interactive. Therefore, investigators should be cautious about interpreting an interaction between factors as an indication that they affect the same process.

The additive factor method generates falsifiable predictions since the effect of prolonging any combination of processes concurrently should be predictable from the effects of prolonging them individually. However, when all the processes are arranged in a sequence the method cannot be used to obtain their order nor their durations.

Not all psychological activities are suitably represented as a sequence of processes arranged end to end. For example, when a subject is given two tasks to perform simultaneously the time required to complete both tasks is usually less than the sum of the times required to perform them separately (Kantowitz, 1974). Evidentially, some of the processes involved in the two tasks can be performed simultancously.

In general a task composed of several processes can be represented as a network in which each arrow represents a process (Fig. 2). Two special types of network are the serial 


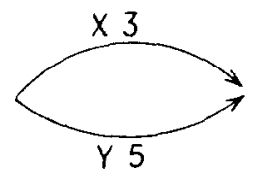

(a)

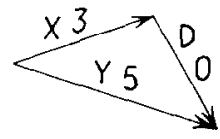

(b)

FIG. 3. (a) A network with $X$ and $Y$ in parallel. (b) A dummy process $D$, with zero duration, rearranges $X$ and $Y$ so they are no longer in parallel.

type of Fig. 1 and the parallel type in Fig. 3. Critical path analysis (Modor \& Phillips, 1970) is a set of techniques for planning and scheduling the processes in such a network. One problem which arises in critical path analysis is calculating the amount of time a task requires for completion given the structure of the network and the durations of the component processes. In studying reaction times we have the inverse problem: We know how much time a task requires for completion under various conditions and we want to know the structure of the task network and the durations of the component processes.

In this paper we use critical path analysis to extend the additive factor method to networks in which the component processes need not be arranged sequentially. From a measurement point of view, we are measuring differences in reaction times to construct a scale of intervals on a network. We find that for a given task we can partially, and sometimes completely, determine the arrangement and ordering of the processes in the network. While we are not able to determine the durations of the processes exactly, we may be able to find intervals within which the durations lie. Finally, we find that verfication of the results of the method need not rest on evidence gathered by other means; measurements made using the method generate predictions which can be verified, or falsified, by other measurements made using the method.

In the first part of the paper we discuss the effects which prolonging processes have on reaction times and the inferences which can be drawn from these effects about the structure of the task network and the durations of the processes. In the second part of the paper we apply our results to a Stroop-like task studied by Greenwald (1972); we find that two bottlenecks can occur when a subject precesses two stimuli cencurrently.

\section{Critical Path Analysis}

We now present some concepts from critical path analysis (Kelley, 1961; Modor \& Phillips, 1970; Weist \& Levy, 1969) and graph theory (Harary, 1969). Some of these concepts were applied to psychological tasks by Christie and Luce (1965). Consider a task composed of a finite number of processes all of which must be completed for the task to be completed. Each process has a starting point, a terminating point, and a duration. It may be that some processes must be completed before others can start.

Such a task may be represented by a network (Fig. 2) in which each process is represented by an arrow directed from the starting point of the process to its terminating point. None of the processes starting at a point may begin until all the processes termina- 
ting at that point are completed. Each arrow has associated with it a nonnegative real number indicating the duration of the corresponding process. The system of lines and points without the numbers is called a graph. Let $G$ denote the graph associated with our task. We use capital letters $X, Y, Z, \ldots$ to refer to processes and small letters $u, v, w, \ldots$ to refer to points. We assume that in $G$ every point is either the starting point or terminating point of some process, i.e., there are no isolated points.

We allow more than one arrow to join two points; we say that two processes $X$ and $Y$ are in parallel if they have the same starting point and the same terminating point. A process with zero duration is called a dummy process; the function of such a process is merely to indicate precedence. One use of dummy arrows by some authors is to rearrange parallel processes so they are no longer in parallel (see Fig. 3); this simplifies notation somewhat. Note that a task can be represented as a network in more than one way.

A path from a point $v_{0}$ to a point $v_{n}$ is an alternating sequence of points and arrows, $v_{0}, X_{1}, v_{1}, X_{2}, \ldots, X_{n}, v_{n}$ in which all the points are distinct and each arrow $X_{i}$ joins the points $\tau_{i-1}$ and $v_{i}$ in that order. $\Lambda$ path may consist of a singlc point. A nontrivial sequence $\varepsilon_{0}, X_{1}, \ldots, v_{0}$ which would be a path except that its first and last points are identical is called a cycle. Paths provide an ordering on the points and arrows of the graph. If a point $u$ preceds a point $v$ on some path we write $u<v$. The same notation is used whenever any point or arrow precedes another point or arrow.

Assumptions about the graph. If there were a cycle in a task network, then the last process in the cycle would have to be completed before the first one could start and vice versa, so we assume that the graph $G$ is acyclic, that is, $G$ has no cycles. For points $u$ and $v$. we write $u \leqq v$ if $u=v v(u$ is the same point as $v$ ) or if $u<v$. The reader can easily verify that in an acyclic graph $\lesssim$ is a partial ordering on points, that is, if $u, v$, and $w$ are points

(i) $u \lesssim u$,

(ii) $u \lesssim v$ and $v \lesssim u$ imply $u=v$,

(iii) if $u \lesssim v$ and $v \lesssim w$ then $u \lesssim w$.

The relation $\lesssim$ defined in a similar way for arrows is also a partial ordering.

Since $G$ has only a finite number of arrows and points and $G$ has no cycles there must be at least onc point which is the terminal point of no arrow. In a psychological task such points can represent the presentation of stimuli which are not preceded by any processes. While there could be several such points, we usually consider only the simplest case in which there is only one such starting point, which we will call $s$. There must also be in $G$ at least one point which is the starting point of no arrow; in a psychological task such points can represent responses which are followed by no further processes. We usually assume we have only one such terminating point, $t$. For every point $u, s \lesssim u \lesssim t$.

Assumptims about the durations. Let $d(X)$ denote the duration of process $X$. The duration of a path is the sum of the durations of all the processes on the path.

Suppose $u \lesssim v$. If all the processes terminating at $u$ have finished, the processes starting at $v$ can begin when and only when all the processes on all the paths between $u$ 
and $v$ are completed, i.e., when and only when all the processes which are on the path of longest duration between $u$ and $v$ are completed. Let $d(u v)$ be the duration of the longest path between $u$ and $v$ (or the duration of any path of maximal duration between $u$ and $v$ if several paths have the same duration and are longer than the others). If there is no path from $u$ to $v$ in $G$ we set $d(u v)=-\infty$. A path of maximal duration between $s$ and $t$ is called a critical path, and its duration $d(s t)$ is the time required to complete the task; in a psychological task, $d(s t)=R T$.

If the terminating point of a process $X$ is the starting point for a process $Y$ then $X$ and $Y$ are said to be adjacent. Two points are said to be adjacent if they are the starting and terminating points of some process. For adjacent points $u$ and $v$ we let $P(u v)$ be the set of all parallel processes starting at $u$ and terminating at $v$. We denote the set of points which are adjacent predecessors of $u$ by $E(u)$ ("entering $u$ ") and we denote the set of points which are adjacent to and follow $u$ by $F(u)$.

The converse graph $G^{\prime}$ of a graph $G$ has the same points as $G$ but with all the arrows directed in the opposite direction. That is, for points $u$ and $v, u \in E(v)$ in $G$ iff $u \in F(v)$ in $G^{\prime}$. The converse of a task graph may have no real world correlate, but we find the concept useful. If $P$ is a path in $G$ then $P^{\prime}$, the corresponding path in the converse $G^{\prime}$, contains all the processes of $P$ but in a different order. Hence the duration of $P^{\prime}$ is the same as the duration of $P$. If $u$ precedes $v$ in $G$ we will let $d^{\prime}(v u)$ be the duration of the longest path between $v$ and $u$ in $G^{\prime}$.

The function $d(u v)$ satisfies the following conditions, which we will call the longest path conditions.

(i) $d(u u)=0$, and if $u \lesssim v$ then $d(u v) \geqslant 0$.

(ii) $d(u v)=d^{\prime}(v u)$.

(iii) If $u \lesssim v \lesssim w$ then $d(u w) \geqslant d(u v)+d(v w)$.

(iv) If $u \in E(v)$ then $d(u v) \geqslant \max \{d(X) \mid X \in P(u v)\}$.

(v) If $u \lesssim v$ then there exists a path $u, X_{1}, p_{1}, X_{2}, \ldots, X_{n}, v$ with

$$
d(u \tau)=\sum_{i=1}^{n} d\left(X_{i}\right)
$$

Note that condition (i) is implied by condition (v). The function $d(u v)$ is similar to a distance function, but is different in some ways. In particular, (iii) is the reverse of the usual triangle inequality. In the kind of task we are considering, all the processes terminating at a point must finish before any process starting at that point can begin. For a task in which a process can start as soon as any process immediately preceding it is completed, we would be concerned with the shortest paths between points rather than the longest ones. The usual triangle inequality holds for the durations of shortest paths. The concepts of critical path analysis would apply to such a task, but the details would have to be modified, of course.

There is an algorithm for calculating $d(u v)$ when $u \lesssim v$ (Modor \& Phillips, 1970). 
We illustrate the algorithm by calculating $d(u v)$ for $u$ and $v$ in the network in Fig. 2, where we have written $d(u x)$ in a circle above each point $x$ between $u$ and $v$. To start we set $d(u u)=0$. Of the two processes between $u$ and $p$ the longer is $B$, so $d(u p)=d(B)=5$. At point $q$ we notice there is only one process, $D$, which is an adjacent predecessor of $q$ and which follows $u ; D$ starts at $p$, so $d(u q)=d(u p)+d(D)=5+1=6$. There are only two processes, $C$ and $E$, which are adjacent predecessors of $v$. Process $C$ can start at time 5 and has duration 7 , hence to reach $v$ via $C$ requires $5+7=d(u p)+d(C)=12$ units of time. Process $E$ can start at 6 and has duration 2, hence to reach $v$ via $E$ requires $6+2=d(u q)+d(E)=8$ units. The time required to reach $v$ via $C$ is the larger time, so $d(u v)=12$.

To find $d(u v)$ for a point $v$ following $u$ we consider all the processes which follow $u$ and immediately precede $v$. We add the duration of each such process to the duration of the longest path from $u$ to the starting point of the process, and we set $d(u v)$ equal to the largest such sum.

The algorithm is summarized in the following two equations. Let $B(u v)$ be the set of all points $i$ such that $u \lesssim i \lesssim v$. Then

(i) $d(u u)=0$

(ii) $d(u v)=\max _{i \in E(v) \cap B(u v)}\left\{d(u i)+\max _{X \in P(i v)}\{d(X)\}\right\}$.

It is not hard to show that Eq. (2) holds if and only if the longest path conditions (iii), (iv), and (v) hold.

Sometimes it is convenient to calculate the duration of the longest path between two points by considering the converse graph. Noting that $i \in E(u)$ in $G^{\prime}$ iff $i \in F(u)$ in $G$, and applying (2) to $G^{\prime}$ we obtain

(i) $d^{\prime}(v v)=0$,

(ii) $d^{\prime}(v u)=\max _{i \in F(v) \cap B(u v)}\left\{d^{\prime}(v i)+\max _{X \in P(u i)}\left\{d^{\prime}(X)\right\}\right\}$.

\section{Slack}

Suppose $X$ precedes $Y$ and that several other processes preceding $Y$ are executed concurrently with $X$ and take a relatively long time to be completed. Then the time when $X$ is completed is not crucial for determining when $Y$ can start. The amount of time by which $X$ can be prolonged without delaying the start of $Y$ is called the slack for $X$ with respect to $Y$, written $S_{X Y}$.

We now derive an expression (Eq. (8)) for slack in terms of the durations of paths in the network. We will see later that reaction times provide information about slacks, indirectly giving us information about path durations via Eq. (8). The reader uninterested in technical details may wish to skim ahead to this equation. Let us denote the starting point of a process $X$ by $x_{1}$ and the terminating point by $x_{2}$. Let $E S_{u}$ denote the earliest starting time, measured from the start of the task, for a point $u$ and let $E S_{X}$ denote the earliest starting time for a process $X$. Recall that $s$ represents the start of the task. Since 
all the processes starting at $x_{1}$ can begin as soon as all the processes preceding $x_{1}$ are finished,

$$
E S_{X}=E S_{x_{1}}=d\left(s x_{1}\right)
$$

Note that $E S_{s}=0$.

Let $E T_{X}$ be the earliest termination time for $X$. Then

$$
E T_{X}=E S_{x_{1}}+d(X)=d\left(s x_{1}\right)+d(X) .
$$

In Fig. 4 the earliest start time for $C$ is 5 and the earliest termination time for $C$ is 9 .

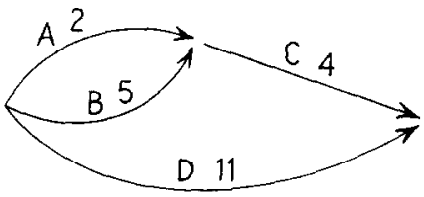

Fig. 4. The slack from $A$ to $C$ is 3 and the slack from $A$ to $t$ is 5 .

Note that $A$ need not be completed at its earliest termination time in order for $C$ to start at its earliest start time. The earliest termination for $A$ is 2 , but $A$ could terminate as late as 5 and not delay the onset of $C$. Let $L T_{X Y}$ be the latest time at which $X$ can terminate and not delay the onset of $Y$. If $Y$ follows $X$ on a path, then, once $X$ is completed, $Y$ can start as soon as all the processes on the longest path between the terminating point of $X$ and the starting point of $Y$ are done. That is,

so

$$
E S_{Y}=L T_{X Y}+d\left(x_{2} y_{1}\right)
$$

$$
L T_{X Y}=d\left(s y_{1}\right)-d\left(x_{2} y_{1}\right)
$$

If no path joins $X$ and $Y$ in $G$ or in $G^{\prime}$ then $d\left(x_{2} y_{1}\right)=-\infty$, so $L T_{X Y}==\infty$, that is, $X$ can terminate at any time and not delay the onset of $Y$. For points $x_{2}$ and $y_{1}$ we write $L T_{x_{2} Y}=L T_{X y_{1}}=L T_{X Y}$ and so on. Note that $L T_{i t}=d(s t)=E S_{t}$.

Let $L S_{X Y}$ be the latest starting time for $X$ which does not delay the start of $Y$. If $Y$ follows $X$ on a path then

$$
L S_{X Y}=L T_{X Y}-d(X)
$$

We write $L S_{X y}=L S_{X Y}$.

The slack for $X$ with respect to $Y$ is equal to the difference between the latest time at which $X$ could terminate without making $Y$ start late and the earliest time at which $X$ could possibly terminate. By (5) and (6) if $X$ precedes $Y$,

$$
\begin{aligned}
S_{X Y} & =L T_{X Y}-E T_{X} \\
& =d\left(s y_{1}\right)-d\left(s x_{1}\right)-d(X)-d\left(x_{2} y_{1}\right) .
\end{aligned}
$$


The slack for $X$ with respect to $Y$ can be written as the difference between the durations of two paths; one is the longest path going from $s$ to the start of $Y$, the other is the longest path containing $X$ going from $s$ to the start of $Y$. If no path joins $X$ and $Y$ then $S_{X Y}=\infty$. We write $S_{X y_{1}}=S_{X Y}$.

As an example, in Fig. $4, S_{A C}=3$.

The amount of time by which the completion of $X$ can be delayed without delaying the completion of the entire task is called the total slack for $X$, written $S_{X t}$. From (8)

$$
S_{X t}=d(s t)-d\left(s x_{1}\right)-d(X)-d\left(x_{2} t\right) .
$$

Using (9) we find $S_{A t}=5$ in Fig. 4.

A process has a total slack of zero if and only if it is on a critical path. Such a process is called a critical process because prolonging it increases the reaction time.

If we think of a point $u$ as a process of zero duration, then from (5) if $u$ precedes $v$

$$
E T_{u}=E S_{u},
$$

and then from (5) and (6)

$$
S_{u v}=L T_{u v}-E T_{u}=d(s v)-d(s u)-d(u v)
$$

It is important not to confuse the slack for $X$ with respect to $y_{1}$ with the slack for $x_{2}$ with respect to $y_{1}$. From (8), (10), and (1) we have $S_{X y_{1}} \geqslant S_{x_{2} y_{1}}$; these two expressions need not be equal.

In $G^{\prime}$, the converse of $G$, let $E S_{u}^{\prime}$ be the earliest start time for $u$ and let $L T_{u s}^{\prime}$ be the latest termination time for $u$ with respect to s. From (4), (6), and (1),

$$
\begin{aligned}
E S_{u}^{\prime} & =d^{\prime}(t u) \\
& =d(s t)-L T_{u t}
\end{aligned}
$$

and

$$
\begin{aligned}
L T_{u s}^{\prime} & =d^{\prime}(t s)-d^{\prime}(u s) \\
& =d(s t)-E S_{u} .
\end{aligned}
$$

If $X$ precedes $Y$ in $G$ we denote the slack from $Y$ to $X$ in $G^{\prime}$ by $S_{Y X}^{\prime}$. We omit the prime from $S_{Y X}^{\prime}$ if the meaning is clear from context.

An example of slack. Welford's $(1952,1967)$ single channel hypothesis provides an example of slack in psychological tasks. Suppose two stimuli are presented, one after the other, and the subject is to make a response to each. We represent the interstimulus interval as a process in the task network (although there may be no corresponding action on the part of the subject). One version of Welford's model assumes that making decisions requires access to a single central channel which can service only one stimulus at a time. If the first stimulus occupies the channel while the second stimulus is presented, the 
processing of the second stimulus must wait until the channel is free. So, according to the model, presentation of the second stimulus can be delayed somewhat without prolonging the time elapsing from the presentation of the first stimulus until the response to the second; that is, the interstimulus interval has slack with respect to the response to the second stimulus.

\section{Analysis of the Task Network: Effects of Prolonging Process}

So far we have assumed that the task under consideration can be represented as a network of partially ordered processes. Each process has a definite point at which it begins, a period during which it is executed, and a definite point at which it stops. There is a point $s$ which precedes every process and every other point. All the processes on every path must be completed, and they must be completed in the order in which they lie on the path. The durations of processes and paths are fixed, not stohastic, and obey the longest path conditions (1).

Now we make a further assumption based on a key idea of Sternberg's (1969a) additive factor method. We assume we are given a set of experimental manipulations which selectively interfere with processes. We assume that each manipulation prolongs the duration of one process, but does not change the duration of any other process, nor does it change the graph. The usefulness of Sternberg's idea of prolonging processes is that "stretching" the arrows leaves the topology of the network intact, facilitating mathematical analysis. We take $d(X)$ as the bascline duration of $X$, so if $X$ is prolonged by $\Delta X$ the new duration for $X$ is $d(X)+\Delta X$. The reaction time $R T=d(s t)$ for the task when all processes are at their baseline levels is considered to be the baseline reaction time; changes in reaction time are measured with respect to $R T$ unless otherwise noted. We use $R T\left(\Delta X_{1}, \ldots, \Delta X_{n}\right)$ to denote the reaction time when $X_{1}, \ldots, X_{n}$ have been prolonged by $\Delta X_{1}, \ldots \Delta X_{n}$, respectively. Other times and time intervals will be denoted analogously.

We use the notation

$$
\begin{aligned}
{[a]^{+} } & =a & & \text { if } \quad a>0 \\
& =0 & & \text { otherwise. }
\end{aligned}
$$

The following identity can easily be proved,

$$
[a]^{+}+\left[b-[a]^{+}\right]^{+}=\max \left\{[a]^{+},[b]^{+}\right\} \text {. }
$$

Suppose process $X$ is prolonged by $\Delta X$. If $X$ is on a critical path then the reaction time is prolonged by $\Delta X$. If $X$ is not on a critical path and $\Delta X \leqslant S_{X t}$ then there is no change in $R T$. And, if $\Delta X>S_{X t}$ then $S_{X t}$ of $\Delta X$ is used to put $X$ on a critical path and the remainder of $\Delta X$ increases $R T$. That is,

$$
R T(\Delta X)=R T+\left[\Delta X^{-}-S_{X t}\right]^{+} .
$$

Let $\Delta R T(\Delta Y)=R T(\Delta X)-R T$. 
Additional details about prolonging a process. (This section is not essential for the main arguments.) The following theorem considers the effect which prolonging one process, $H$, has on another process, $C$.

Theorem 1. Let $H$ and $C$ be two processes.

(a) If $H$ does not precede $C$,

$$
E S_{C}(\Delta H)=E S_{C}
$$

(b) If $C$ does not precede $H$,

$$
L T_{C t}(\Delta H)-L T_{C t}+\left[\Delta H-S_{H t}\right]^{+} .
$$

(c) If $H$ precedes $C$,

$$
E S_{C}(\Delta H)=E S_{C}+\left[\Delta H-S_{H C}\right]^{+} .
$$

(d) If $C$ precedes $H$,

$$
L T_{C t}(\Delta H)=L T_{C t}+\left[\Delta H-S_{H t}\right]^{+}-\left[\Delta H-S_{H C}^{\prime}\right]^{+} .
$$

Proof. (a) For a process $A$ let $\mu(A)$ be the largest number of processes on any path between $s$ and $a_{1}$. Suppose $C$ is a process not preceded by $H$ for which $\mu(C)=0$. Then $c_{1}=s$ and $E S_{C}(\Delta H)=E S_{s}=E S_{C}$.

Suppose for all processes $A$ not preceded by $I I$ if $\mu(A)<n$ then $E S_{A}(\Delta I I)=E S_{A}$. Suppose $C$ is a process not preceded by $H$ with $\mu(C)=n$. Then, by (2) and (4), noting that $E\left(c_{1}\right) \subset B\left(s c_{1}\right)$,

$$
\begin{aligned}
E S_{C}(\Delta H) & =\max _{i \in E\left(c_{1}\right)}\left\{E S_{i}(\Delta H)+\max _{\left.X \in P_{i\left(i c_{1}\right.}\right)}\{d(X)\}\right\} \\
& =\max _{i \in E^{\prime}\left(c_{1}\right)}\left\{E S_{i}+\max _{X \in P^{\prime}\left(i c_{1}\right)}\{d(X)\}\right\} \\
& =E S_{C} .
\end{aligned}
$$

(b) For a process $A$ let $\lambda(A)$ be the largest number of processes on any path from $a_{2}$ to $t$. Suppose $C$ is a process not preceding $H$ for which $\lambda(C)=0$. Then $c_{2}=t$ and since $L T_{t t}=R T$, by (14)

$$
L T_{c_{2}}(\Delta H)=L T_{t t}(\Delta H)=L T_{t t}+\left[\Delta H-S_{H t}\right]^{+} .
$$

Suppose for all processes $A$ not preceding $H$, if $\lambda(A)<n$ then $L T_{A t}(\Delta I I)=L T_{A t}+$ $\left[\Delta H-S_{H t}\right]^{+}$. Suppose $C$ is a process not preceding $H$ for which $\lambda(C)=n$. We first derive an expression for $L T_{C t}$ when $H$ is not prolonged. 
By (6) and (3), noting that $F\left(c_{2}\right) \subset B\left(c_{2} t\right)$,

$$
\begin{aligned}
L T_{C t} & =d(s t)-d\left(c_{2} t\right) \\
& =d(s t)-\max _{i \in F\left(c_{2}\right)}\left\{d(i t)+\max _{X \in P\left(c_{2} i\right)}\{d(X)\}\right\} \\
& =\min _{i \in F\left(c_{2}\right)}\left\{d(s t)-d(i t)-\max _{X \in P\left(c_{2} i\right)}\{d(X)\}\right\} \\
& =\min _{i \in F\left(c_{2}\right)}\left\{L T_{i t}-\max _{X \in P\left(c_{2} i\right)}\{d(X)\}\right\} .
\end{aligned}
$$

If we prolong $H$ by $\Delta H$ the above expression becomes

$$
L T_{C t}(\Delta H)=\min _{i \in F\left(c_{2}\right)}\left\{L T_{i t}(\Delta H)-\max _{X \in P\left(c_{2} i\right)}\{d(X)\}\right\} .
$$

By the induction hypothesis,

$$
\begin{aligned}
L T_{C t}(\Delta H) & =\min _{i \in F\left(c_{2}\right)}\left\{L T_{i t}-\max _{X \in P\left(c_{2} i\right)}\{d(X)\}\right\}+\left[\Delta H-S_{H t}\right]^{+} \\
& =L T_{C t}+\left[\Delta I I-S_{H t}\right]^{+} .
\end{aligned}
$$

(c) By (2) and (4) if we remove all processes not preceding $C$ from the network $E S_{C}$ is not changed. In the remaining subnetwork $c_{1}$ is the terminus since every process precedes $c_{1}$ and $c_{1}$ precedes no process. Let $E S_{c_{1}}^{*}$ and $L T_{c_{1}}^{*}$ be the earliest start time and latest termination time for $c_{1}$ in the remaining subnetwork. Using (b) of this theorem,

$$
\begin{aligned}
E S_{c_{1}}(\Delta H) & =E S_{c_{1}}^{*}(\Delta H)=L T_{c_{1} c_{1}}^{*}(\Delta H) \\
& =L T_{c_{1_{1} c_{1}}}^{*}+\left[\Delta H-S_{H C}\right]^{+} \\
& =E S_{c_{1}}+\left[\Delta H-S_{H C}\right]^{+}
\end{aligned}
$$

(d) If $C$ precedes $H$ then in the converse graph $H$ precedes $C$. Then by (c) of this theorem

$$
E S_{c_{2}}^{\prime}(\Delta H)=E S_{c_{2}}^{\prime}+\left[\Delta H-S_{H c_{2}}^{\prime}\right]^{+}
$$

By (11), (14), and (6)

$$
\begin{aligned}
L T_{c_{2} t}(\Delta I I) & =R T(\Delta I I)-E S_{c_{2}}^{\prime}(\Delta I I) \\
& =L T_{c_{2} t}+\left[\Delta H-S_{H t}\right]^{+}-\left[\Delta H-S_{H C}^{\prime}\right]^{+}
\end{aligned}
$$

\section{Effects of Prolonging Two Processes}

Our goal in this section is to describe what happens to reaction times when two processes are prolonged. Theorem 2 below shows that the effects depend on whether the two processes are on a path together or not. 
This problem was investigated in another context by Shapley (1959) who derived equations for the maximal flow through a network as a function of the capacities of two arrows (Ford \& Fulkerson, 1962).

Let $d_{\Delta X}(u v)$ be the duration of the longest path between $u$ and $v$ when $X$ is prolonged by $\Delta X$. We say that a process $X$ is between points $u$ and $v$ if $u<X<v$. From (1v) it is clear that if $X$ is not between $u$ and $v$ then prolonging $X$ does not change the duration of the longest path between $u$ and $v$, that is,

$$
d_{\Delta X}(u v)=d(u v) .
$$

Prolonging one process may change the total slack available to another process. If prolonging $X$ delays the termination of the task, then a process $Y$ not on a path with $X$ does not have to be finished as soon as usual; $Y$ may gain some extra total slack. Let $S_{Y t}(\Delta X)$ be the total slack for $Y$ when $X$ is prolonged by $\Delta X$ and suppose $X$ is not on a path with $Y$. Then by equations (9), (19), and the assumption of selective inference

$$
\begin{aligned}
S_{Y t}(\Delta X) & =d_{\Delta X}(s t)-d_{\Delta X}\left(s y_{1}\right)-d_{\Delta X}(Y)-d_{\Delta X}\left(y_{2} t\right) \\
& =d_{\Delta X}(s t)-d(s t)+d(s t)-d\left(s y_{1}\right)-d(Y)-d\left(y_{2} t\right) .
\end{aligned}
$$

Then since by (14), $d_{\Delta X}(s t)-d(s t)=\left[\Delta X-S_{X t}\right]^{+}$, we have

$$
S_{Y t}(\Delta X)=S_{Y t}+\left[\Delta X-S_{X t}\right]^{+} .
$$

(Equation (24) below is the analogous expression for the case when $X$ precedes $Y$.)

The following theorem is our basic tool for analyzing the task network. When Sternberg's method is extended to tasks in which some processes are executed concurrently, the effect of prolonging both $X$ and $Y$ need not be the sum of the effects of prolonging them individually. Sternberg (1969a) pointed out that the effect of prolonging both of two processes in parallel would be the maximum of the effects of prolonging them individually. The theorem shows that this result is true in general for any two processes not joined by a path. Even if $X$ and $Y$ are on a path together, the effect of prolonging both need not be the sum of the effects of prolonging them individually. Investigators should be cautious about interpreting an interaction between two factors as an indication that the two factors influence the same process. Such interactions will be commonly found in tasks in which the mental processes are not arranged in a sequence of stages.

Theorem 2. Suppose $X$ is prolonged by $\Delta X$ and $Y$ by $\Delta Y$.

(a) If no path joins $X$ and $Y$ then

$$
\Delta R T(\Delta X, \Delta Y)=\max \{\Delta R T(\Delta X), \Delta R T(\Delta Y)\} .
$$

(b) Suppose $X$ precedes $Y$ and $\Delta X$ and $\Delta Y$ are large enough so that $\Delta R T(\Delta X)$, $\Delta R T(\Delta Y)>0$ and so that when $X$ is prolonged by $\Delta X$ and $Y$ by $\Delta Y$ there is a critical path containing both $X$ and $Y$. 
Then

$$
\Delta R T(\Delta X, \Delta Y)=\Delta R T(\Delta X)+\Delta R T(\Delta Y)+K(X Y),
$$

where $K(X Y)=S_{X t}-S_{X Y}$.

Proof. (a) If we consider $R T(\Delta X, \Delta Y)$ to be $R T(\Delta X)$ plus the change in $R T(\Delta X)$ produced by prolonging $Y$ by $\Delta Y$, then by (14) and (20),

$$
\begin{aligned}
R T(\Delta X, \Delta Y) & =R T(\Delta X)+\left[\Delta Y-S_{Y t}(\Delta X)\right]^{+} \\
& =R T+\left[\Delta X-S_{X i}\right]^{+}+\left[\Delta Y-S_{Y t}-\left[\Delta X-S_{X t}\right]^{+}\right]^{+} .
\end{aligned}
$$

Then, using identity (13),

$$
\Delta R T(\Delta X, \Delta Y)=\max \left\{\left[\Delta X-S_{X t}\right]^{+},\left[\Delta Y-S_{Y t}\right]^{+}\right\} .
$$

(b) By Eqs. (8) and (9)

and

$$
d(s t)-d\left(s y_{1}\right)=d(Y)+d\left(y_{2} t\right)+S_{Y t}
$$

$$
d\left(s y_{1}\right)=d\left(s x_{1}\right)+d(X)+d\left(x_{2} y_{1}\right)+S_{X Y} .
$$

By addition,

$$
R T=d(s t)=d\left(s x_{1}\right)+d(X)+d\left(x_{2} y_{1}\right)+S_{X Y}+d(Y)+d\left(y_{2} t\right)+S_{Y t} .
$$

When $X$ is prolonged by $\Delta X$ and $Y$ by $\Delta Y$ a critical path passes through both $X$ and $Y$. Then the reaction time is equal to the duration of the longest path going from $s$ to $x_{1}$, along $X$, from $x_{2}$ to $y_{1}$, along $Y$ and from $y_{2}$ to $t$. Then by (19) and the assumption of selective interference,

$$
R T(\Delta X, \Delta Y)=d\left(s x_{1}\right)+d(X)+\Delta X+d\left(x_{2} y_{1}\right)+d(Y)+\Delta Y+d\left(y_{2} t\right) .
$$

Subtracting the above two equations we obtain

$$
\Delta R T(\Delta X, \Delta Y)=\Delta X-S_{X Y}+\Delta Y-S_{Y t} .
$$

Then by (14),

$$
\Delta R T(\Delta X, \Delta Y)=\Delta R T(\Delta X)+S_{X t}-S_{X Y}+\Delta R T(\Delta Y) .
$$

We call $K(X Y)$ the coupled slack between $X$ and $Y$. It is the synergistic effect on reaction timc of prolonging $X$ and $Y$ together. The magnitude of $K(X Y)$ can be determined empirically by calculating the difference between the effect of prolonging $X$ and $Y$ simultaneously and the sum of the effects of prolonging them individually, for prolongations which are not too small.

By (8) and (9),

$$
K(X Y)=d(s t)-d\left(s y_{1}\right)-d\left(x_{2} t\right)+d\left(x_{2} y_{1}\right)
$$


We will see as we go that coupled slack provides information about the scheduling and durations of processes.

Note that the value of $K(X Y)$ is independent of $\Delta X$ and $\Delta Y$. If several levels of prolongations are used and $\Delta R T(\Delta X, \Delta Y)-\Delta R T(\Delta X)-\Delta R T(\Delta Y)$ changes with the levels of $\Delta X$ and $\Delta Y$ then Theorem $2(\mathrm{~b})$ does not hold for the experiment. This is the first of three tests of the validity of the method which we point out.

As an example of the effect of coupled slack on reaction time, consider Fig. 4. Equation (22) applies to processes $A$ and $C$. If we prolong $A$ by $\Delta A=7$ the new reaction time is 13, so $\Delta R T(\Delta A)=13-11=2$. If we prolong $C$ by $\Delta C=6$ the new reaction time is 15 , so $\Delta R T(\Delta C)=4$. Now suppose we prolong $A$ by 7 and at the same time prolong $C$ by 6 . The resulting $R T$ is 19 , so $\Delta R T(\Delta A, \Delta C)=8$. We have calculated above that $S_{A t}=5$ and $S_{A C}=3$, so $K(A C)=2$. Hence,

$$
\Delta R T(\Delta A, \Delta C)=\Delta R T(\Delta A)+\Delta R T(\Delta C)+2 .
$$

Note that increasing $\triangle A$ and $\triangle C$ leaves the value of $K(A C)$ unchanged at 2. No path joins $A$ and $B$ in Fig. 4 and Eq. (21) holds for them. The coupled slack for $A$ and $B$ is not a real number; by Eq. (23) the coupled slack for two processes not joined by a path is negative infinity.

Coupled slack can be positive, as in the example we just gave, but it can also be zero or, as we will see later, negative. If all the processes are arranged in a sequence, as in Fig. 1, then all slacks are zero and $K(X Y)=0$ for every pair of processes $X$ and $Y$. Then (22) becomes

$$
\Delta R T(\Delta X, \Delta Y)=\Delta R T(\Delta X)+\Delta R T(\Delta Y),
$$

that is, the usual additive factor situation is a special case for which (22) holds.

If $X$ and $Y$ are on a path together the value of $K(X Y)$ provides no information about which comes first on the path. Suppose $X$ comes before $Y$ in the task and consider the converse network in which $Y$ comes before $X$. Let $K^{\prime}(Y X)$ be the coupled slack in the converse network. Applying (8) and (9) to the converse we obtain

$$
S_{Y_{s}}^{\prime}=d^{\prime}(t s)-d^{\prime}\left(t y_{2}\right)-d(Y)-d^{\prime}\left(y_{1} s\right)
$$

and

$$
S_{Y X}^{\prime}=d^{\prime}\left(t x_{2}\right)-d^{\prime}\left(t y_{2}\right)-d(Y)-d\left(y_{1} x_{2}\right) .
$$

Subtracting and using the fact that $d(u v)=d^{\prime}(v u)$ we find

$$
\begin{aligned}
K^{\prime}(X Y) & =S_{Y s}^{\prime}-S_{Y X}^{\prime} \\
& =d(s t)-d\left(s y_{1}\right)-d\left(x_{2} t\right)+d\left(x_{2} y_{1}\right) \\
& =K(X Y) .
\end{aligned}
$$

Therefore, $K(X Y)$ alone cannot be used to determine the precedence of $X$ and $Y$. Incidentally, we have shown that $S_{Y t}-S_{Y X}^{\prime}=S_{X t}-S_{X Y}$. 
Small prolongations of processes on a path. What happens if $X$ and $Y$ are on a path together but their prolongations are too small for Theorem 2 to apply? The answer turns out to be somewhat complicated.

Let us begin by considering the change in the total slack for $Y$ if $X$ precedes $Y$ and is prolonged by $\Delta X$. By (8) applied to $S_{Y t}$ and Theorem 1(b) and (c),

$$
\begin{aligned}
S_{Y t}(\Delta X) & =L T_{Y t}(\Delta X)-E T_{Y}(\Delta X) \\
& =L T_{Y t}+\left[\Delta X-S_{X t}\right]^{+}-E T_{Y}-\left[\Delta X-S_{X Y}\right]^{+} \\
& =S_{Y t}+\left[\Delta X-S_{X t}\right]^{+}-\left[\Delta X-S_{X Y}\right]^{+} .
\end{aligned}
$$

(This equation is analogous to (20) for $X$ and $Y$ not on a path together.)

We now derive the general expression for the change in $R T$ when $X$ and $Y$ are prolonged and on a path together. Suppose $X$ precedes $Y$ and $X$ is prolonged by $\Delta X$ and $Y$ by $\Delta Y$. Then from (14) and (24)

$$
\begin{aligned}
R T(\Delta X, \Delta Y) & =R T(\Delta X)+\left[\Delta Y-S_{Y t}(\Delta X)\right]^{+} \\
& =R T+\left[\Delta X-S_{X t}\right]^{+}+\left[\Delta Y-S_{Y t}-\left[\Delta X-S_{X t}\right]^{+}+\left[\Delta X-S_{X Y}\right]^{+}\right]^{+} .
\end{aligned}
$$

Then by identity (13),

$$
\Delta R T(\Delta X, \Delta Y)=\max \left\{\left[\Delta X-S_{X t}\right]^{+},\left[\Delta Y-S_{Y t}+\left[\Delta X-S_{X Y}\right]^{+}\right]^{+}\right\} .
$$

Note that when the assumptions of Theorem 2(b) hold all the bracketed expressions in (25) are positive and so $\Delta R T(\Delta X, \Delta Y)=\Delta R T(\Delta X)+\Delta R T(\Delta Y)+K(X Y)$.

Since any pair of processes are either on a path together or not, the only two kinds of interaction consistent with our method are those of form (21) or (26). We have nothing to say about interactions of other forms; such interactions indicate, of course, that some of our assumptions have been violated. For a discussion of interactions not covered by (21) or (26) but which were used to infer that the processing in a task was not done in a sequence of stages arranged end to end, the reader is referred to Miller (1976).

Negative coupled slack. Suppose $X$ and $Y$ are joined by a path, but $S_{X t}<S_{X Y}$. Then $K(X Y)$ is negative. If $S_{X t}<\Delta X<S_{X Y}$ or if $S_{Y t}<\Delta Y<S_{Y X}$, then from (26), cren though $X$ and $Y$ are joined by a path

$$
\Delta R T(\Delta X, \Delta Y)=\max \left\{\left[\Delta X-S_{X t}\right]^{+},\left[\Delta Y-S_{Y t}\right]^{+}\right\},
$$

which is the same as Eq. (21) for $X$ and $Y$ not joined by a path. If $\Delta X$ or $\Delta Y$ is small and $K(X Y)<0$ then $X$ and $Y$ may mimic the behavior of processes not joined by a path.

Townsend $(1971,1976)$ has discovered a fundamental limitation in analyzing reaction times. Processes in serial are generally indistinguishable form processes in parallel on the basis of the distributions of their completion times. Equations (21) and (22) overcome this limitation to some extent by considering processes which are prolonged. Nevertheless, Townsend's problem of indeterminacy arises with prolonged processes; 
small prolongations cannot distinguish processes on a path together with negative coupled slack from processes not on a path together at all.

How can we distinguish these cases empirically? A sure method is to use long prolongations, because Eq. (21) and (26) behave differently as the levels of prolongations are increased. If $\Delta X$ and $\Delta Y$ are so large that $\Delta R T(\Delta X), \Delta R T(\Delta Y)>R T$, then if $X$ and $Y$ are joined by a path Eq. (22) should hold. So if Eq. (21) holds for such long prolongations one may conclude that $X$ and $Y$ are not joined by a path. Unfortunately, no simple method exists for distinguishing these two cases and the difficulties are accentuated by the stochastic nature of reaction times.

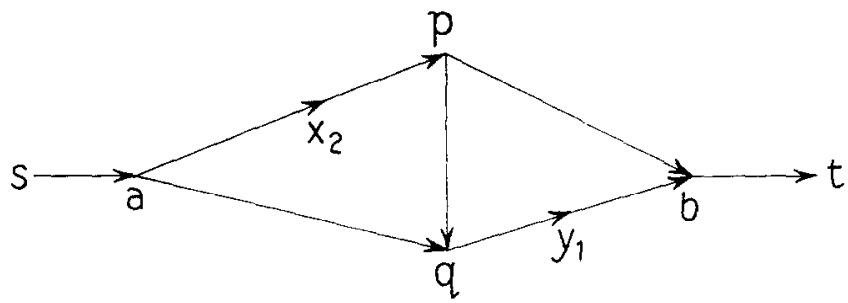

FIG. 5. If the coupled slack between $X$ and $Y$ is a negative real number then the task network contains a Wheatstone bridge shaped subnetwork; $s$ may coincide with $a$ and $t$ with $b$.

The following theorem shows that if $K(X Y)<0$ the task graph must contain the Wheatstone bridge shaped subgraph illustrated in Fig. 5 (or the converse subgraph). Note that it is not the shape of the network alone which yields negative coupled slack; for some durations of the paths, $X$ and $Y$ can be arranged in such a subgraph with $K(X Y) \geqslant 0$.

Theorem 3. Suppose $X$ precedes $Y$. Then $K(X Y)<0$ iff all of the following conditions hold.

(i) The longest path from $x_{2}$ to $y_{1}$ is not contained in the longest path from $x_{2}$ to $t$; we can let $p$ be the last point preceding $y_{1}$ to be on both paths;

(ii) the longest path from $x_{2}$ to $y_{1}$ is not contained in the longest path from s to $y_{1}$; we can let $q$ be the first point following $x_{2}$ to be on both paths;

(iii) $p \neq q$ and

$$
d(s t)<d(s q)+d(p t)-d(p q)
$$

Proof. Suppose $0>K(X Y)=d(s t)-d\left(s y_{1}\right)-d\left(x_{2} t\right)+d\left(x_{2} y_{1}\right)$. If $y_{1}$ were on the longest path from $x_{2}$ to $t$, then $d\left(x_{2} t\right)=d\left(x_{2} y_{1}\right)+d\left(y_{1} t\right)$ and by substitution

$$
K(X Y)=d(s t)-d\left(s y_{1}\right)-d\left(y_{1} t\right) \geqslant 0,
$$

a contradiction. Likewise, $x_{2}$ is not on the longest path from $s$ to $y_{1}$. 
Choose $p$ and $q$ as stated, then

and

$$
\begin{aligned}
& d\left(s y_{1}\right)=d(s q)+d\left(q y_{1}\right), \\
& d\left(x_{2} t\right)=d\left(x_{2} p\right)+d(p t),
\end{aligned}
$$

$$
d\left(x_{2} y_{1}\right)=d\left(x_{2} p\right)+d(p q)+d\left(q y_{1}\right)
$$

Substituting these expressions into the equation for $K(X Y)$ yields

$$
K(X Y)=d(s t)-d(s q)-d(p t)+d(p q)<0
$$

and statement (iii) follows immediately.

If (i), (ii), and (iii) hold then clearly $K(X Y)<0$.

\begin{tabular}{|c|c|c|c|c|}
\hline \multicolumn{5}{|c|}{$K(X Y)=S_{X t}-S_{X Y} \geqslant 0$} \\
\hline$\Delta X$ & $\Delta Y$ & $\Delta R T(\Delta X)$ & $\Delta R T(\Delta Y)$ & $\Delta R T(\Delta X, \Delta Y)$ \\
\hline$\Delta X \leqslant S_{X Y} \leqslant S_{X !}$ & $\Delta Y \leqslant S_{Y X}^{\prime} \leqslant S_{Y t}$ & 0 & 0 & 0 \\
\hline$S_{X Y} \leqslant \Delta X \leqslant S_{X t}$ & $\Delta Y \leqslant S_{Y X}^{\prime} \leqslant S_{Y t}$ & 0 & 0 & 0 \\
\hline$S_{X Y} \leqslant S_{X t} \leqslant \Delta X$ & $\Delta Y \leqslant S_{Y X}^{\prime} \leqslant S_{Y t}$ & $\Delta X-S_{X !}$ & 0 & $\Delta X-S_{X \prime}$ \\
\hline$\Delta X \leqslant S_{X Y} \leqslant S_{X t}$ & $S_{Y X}^{\prime} \leqslant \Delta Y \leqslant S_{Y t}$ & 0 & 0 & 0 \\
\hline$S_{X Y} \leqslant \Delta X \leqslant S_{X t}$ & $S_{Y X}^{\prime} \leqslant \Delta Y \leqslant S_{Y t}$ & $\mathbf{0}$ & 0 & {$\left[\Delta Y-S_{Y t} \div \Delta X-S_{X Y}\right]^{+}$} \\
\hline$S_{X Y} \leqslant S_{X t} \leqslant \Delta X$ & $S_{Y X}^{\prime} \leqslant \Delta Y \leqslant S_{Y t}$ & $\Delta X-S_{X t}$ & 0 & $\Delta X-S_{X t}+\Delta Y-S_{Y X}^{\prime}$ \\
\hline$\Delta X \leqslant S_{X Y} \leqslant S_{X t}$ & $S_{Y X}^{\prime} \leqslant S_{Y t} \leqslant \Delta Y$ & $\mathbf{0}$ & $\Delta Y-S_{Y t}$ & $\Delta Y^{\prime}-S_{Y_{t}}$ \\
\hline$S_{X Y} \leqslant \Delta X \leqslant S_{X t}$ & $S_{Y X}^{\prime} \leqslant S_{Y t} \leqslant \Delta Y$ & $\mathbf{0}$ & $\Delta Y-S_{Y t}$ & $\Delta Y-S_{Y t}+\Delta X-S_{X Y}$ \\
\hline$S_{X Y} \leqslant S_{X t} \leqslant \Delta X$ & $S_{Y X}^{\prime} \leqslant S_{Y t} \leqslant \Delta Y$ & $\Delta X-S_{X t}$ & $\Delta Y-S_{Y^{t}}$ & $\Delta X-S_{X !}+\Delta Y-S_{Y t}+K_{X Y}$ \\
\hline \multicolumn{5}{|c|}{$K(X Y)=S_{X t}-S_{X Y} \leqslant 0$} \\
\hline$\Delta X$ & $\Delta Y$ & $\Delta R T(\Delta X)$ & $\Delta R T(\Delta Y)$ & $\Delta R T(\Delta X, \Delta Y)$ \\
\hline$\Delta X \leqslant S_{X i} \leqslant S_{X Y}$ & $\Delta Y \leqslant S_{Y !} \leqslant S_{Y X}^{\prime}$ & 0 & 0 & 0 \\
\hline$S_{X \ell} \leqslant \Delta X \leqslant S_{X Y}$ & $\Delta Y \leqslant S_{Y !} \leqslant S_{Y X}^{\prime}$ & $\Delta X-S_{X t}$ & 0 & $\Delta X-S_{X t}$ \\
\hline$S_{X t} \leqslant S_{X Y} \leqslant \Delta X$ & $\Delta Y \leqslant S_{Y} \leqslant S_{Y X}^{\prime}$ & $\Delta X-S_{X I}$ & 0 & $\Delta X-S_{X t}$ \\
\hline$\Delta X \leqslant S_{X t} \leqslant S_{X Y}$ & $S_{Y \prime} \leqslant \Delta Y \leqslant S_{Y X}^{\prime}$ & 0 & $\Delta Y^{T}-S_{Y t}$ & $\Delta Y-S_{Y \prime}$ \\
\hline$S_{X \prime} \leqslant \Delta X \leqslant S_{X Y}$ & $S_{Y t} \leqslant \Delta Y \leqslant S_{Y X}^{\prime}$ & $\Delta X-S_{X i}$ & $\Delta Y-S_{Y t}$ & $\max \left\{\Delta X-S_{X l}, \Delta Y-S_{Y+\}}\right.$ \\
\hline$S_{X t} \leqslant S_{X Y} \leqslant \Delta X$ & $S_{Y \mathrm{t}} \leqslant \Delta Y \leqslant S_{Y X}^{\prime}$ & $\Delta X-S_{X t}$ & $\Delta Y-S_{Y t}$ & $\Delta X-S_{X t}$ \\
\hline$\Delta X \leqslant S_{X t} \leqslant S_{X Y}$ & $S_{Y t} \leqslant S_{Y X}^{*} \leqslant \Delta Y$ & 0 & $\Delta Y-S_{Y t}$ & $\Delta Y-S_{Y t}$ \\
\hline$S_{X \prime} \leqslant \Delta X \leqslant S_{X Y}$ & $S_{Y \ell} \leqslant S_{Y X}^{\prime} \leqslant \Delta Y$ & $\Delta X-S_{X t}$ & $\Delta Y-S_{Y_{t}}$ & $\Delta Y-S_{Y t}$ \\
\hline$S_{X \prime} \leqslant S_{X Y} \leqslant \Delta X$ & $S_{Y t} \leqslant S_{Y X}^{\prime} \leqslant \Delta Y$ & $\Delta X-S_{X t}$ & $\Delta Y-S_{Y t}$ & $\Delta X-S_{X t}+\Delta Y$ \\
\hline
\end{tabular}

Q.E.D.

IABLE 1

$\Delta R T(\Delta X, \Delta Y)$ as a Function of $\Delta X$ and $\Delta Y$ in Eq. (26) for $X$ Preceding $Y$ 
Because Eq. (26) is a complicated expression, we have displayed in Table 1 the value of $\Delta R T(\Delta X, \Delta Y)$ as a function of $\Delta X$ and $\Delta Y$ when (26) holds.

\section{Stochastic Networks}

We have assumed that a process $X$ always requires the same amount of time $d(X)$, and that if $X$ is prolonged it is always prolonged by a fixed amount $\Delta X$. In practice the durations and prolongations are likely to be random variables, creating a problem in applying the method to psychological data.

Unfortunately, the statistical problems involved in using the critical path method seem to be formidable. The interested reader is referred to Fulkerson (1962) and Sielken et al. (1974) for approaches to these problems. The crux of the difficulty is in equations such as (1), (21), and (26) which involve the maximum of two random variables in the stochastic situation. Finding the distribution of $Z=\max \{X, Y\}$, where $X$ and $Y$ are random variables is straightforward if $X$ and $Y$ are independent and identically distributed, but may be almost impossible if they are not.

There is no problem when prolongations are long and processes are on a path together. Suppose $Y$ and $Y$ are on a path together with say, $X$ preceeding $Y$. Suppose $\Delta X, \Delta Y$, and the path durations are random variables. If on every trial the prolongations are so long that $\Delta X \geqslant S_{X t}, S_{X Y}$ and $\Delta Y \geqslant S_{Y t}, S_{Y X}^{\prime}$, then by taking expected values in Eq. (22) we obtain

$$
E \Delta R T(\Delta X, \Delta Y)=E \Delta R T(\Delta X)+E \Delta R T(\Delta Y)+E K(X Y),
$$

where $E Z$ denotes the expected value of a random variable $Z$. The tests of validity discussed in this paper turn out to be applicable to the expected values of the variables involved when the processes are on a path together and their prolongations are large.

However, a problem arises in trying to use small prolongations to distinguish the case in which $X$ and $Y$ are not on a path together from the case in which they are on a path together with negative coupled slack.

In the deterministic case, if

$$
\max \{\Delta R T(\Delta X), \Delta R T(\Delta Y)\}<\Delta R T(\Delta X, \Delta Y)<\Delta R T(\Delta X)+\Delta R T(\Delta Y)
$$

then from Table 1 we could conclude that $X$ and $Y$ are in a Wheatstone bridge. But since

$$
\begin{aligned}
\max \{E \Delta R T(\Delta X), E \Delta R T(\Delta Y)\} \leqslant & E \max \{\Delta R T(\Delta X), \Delta R T(\Delta Y)\} \leqslant E R T(\Delta X) \\
& +E \Delta R T(\Delta Y)
\end{aligned}
$$

it may often be the case that (27) holds for the expected values of the changes in reaction time, even though $X$ and $Y$ are not on a path together. In the deterministic case processes in a Wheatstone bridge can mimic processes not on a path together. This mimicking is facilitated by stochastic processing times. Further research on these problems is clearly needed. 


\section{Measurements of Time at Several Points}

We have assumed so far that time is recorded at only two points, $s$ and $t$, but we may be able to record time at several points in the network. We may know the times at which various stimuli are presented and responses made, and we may also know the times at which various physiological events occur. We will see that measurements of time at several points can provide valuable information about the order and durations of the processes in the network; furthermore, we can check such measurements against each other in order to verify our assumptions.

A point of the network not preceded by any other point is called a source and a point not followed by any other point is called a sink. If there are several sources and several sinks in the network, we can add a new point $s$ preceding each source and a new point $t$ following each sink; in the resulting network $s \lesssim u \lesssim t$ for every point $u$ and our previous results can be applied in a straightforward way.

Consider a network with a single source $s$ and suppose we can measure the reaction time $R T_{u}$ from $s$ to a point $u$. If all the processes start as soon as possible then $R T_{u}=E S_{u}$. Consider a new network formed by removing from the task network all points and processes which do not precede $u$. Suppose $X<Y<u$. Note that the slack for $X$ with respect to $Y$ is independent of $u$. Let

$$
K_{u}(X Y)=S_{X u}-S_{X Y} \cdot
$$

If $\Delta X$ and $\Delta Y$ are large enough so that Theorem 2(b) applies to the new network, then

$$
\Delta R T_{u}(\Delta X, \Delta Y)=\Delta R T_{u}(\Delta X)+\Delta R T_{u}(\Delta Y)+K_{u}(X Y) .
$$

Let $S_{Y X}^{\prime}(u)$ be the slack for $Y$ with respect to $X$ in the converse of the new network. Note that for two points $u$ and $v$ with $X<Y<u$, $v$ it need not be true that $S_{Y X}^{\prime}(u)$ equals $S_{Y X}^{\prime}(v)$.

The next theorem presents two simple but important relationships which hold for reaction times measured at two points.

'THeorem 4. Let process $X$ precede process $Y$ which precedes points $u$ and $v$.

(a) If $\Delta R T_{u}(\Delta X)$ and $\Delta R T_{v}(\Delta X)$ are both positive, then

$$
\Delta R T_{u}(\Delta X)+K_{u}(X Y)=\Delta R T_{v}(\Delta X)+K_{v}(X Y) .
$$

(b) If $\Delta Y$ is greater than $S_{Y^{r} u}, S_{Y v}, S_{Y X}^{\prime}(u)$, and $S_{Y X}^{\prime}(v)$, then

$$
\Delta R T_{u}(\Delta X, \Delta Y)-\Delta R T_{u}(\Delta Y)=\Delta R T_{v}(\Delta X, \Delta Y)-\Delta R T_{v}(\Delta Y) .
$$

To apply Theorem 4(b) we must know whether the hypotheses hold; this can sometimes be inferred from the data by using Table 1 . If $\Delta X$ and $\Delta Y$ are both relatively large then, of course, parts (a) and (b) are equivalent. The proof of (a) follows from (14) and the definition of coupled slack. The proof of (b) follows immediately from (26) applied to $\Delta R T_{u}(\Delta X, \Delta Y)$ and $\Delta R T_{v}(\Delta X, \Delta Y)$, see Table 1. 
If $\Delta R T_{v}(\Delta X)$ and $\Delta R T_{u}(\Delta X)$ are both positive, then the durations of the longest path between $x_{2}$ and $u$ and the longest path between $x_{2}$ and $v$ are related by

$$
R T_{u}(\Delta X)-R T_{v}(\Delta X)=d\left(x_{2} u\right)-d\left(x_{2} v\right)
$$

the derivation is straightforward. Another equation which is useful because it provides both a means of verifying our conclusions and of ordering the processes in the network is the following. If $s$ precedes $X, Y$, and $Z$, which precede $u$ and $v$, and if furthermore $X<Y<Z$, then

$$
K_{u}(X Z)-K_{u}(X Y)=K_{v}(X Z)-K_{v}(X Y)
$$

again the derivation is straightforward.

Theorem 4 is important for two reasons. First, it can be used to determine the order of two processes. When $X$ precedes $Y$ the equations of the theorem will not hold if expressions in $X$ are substituted for the corresponding expressions in $Y$ and vice versa (unless $S_{Y X}^{\prime}(u)$ happens to equal $S_{Y X}^{\prime}(v)$ or both sides in $(b)$ are 0 ). Second, the equations are independent of equations of form (22) and thus provide a test of the applicability of the method. This is the second of the three tests of the method which we discuss in this paper.

\section{Prolonging Several Processes.}

The results of prolonging several processes can, in general, be predicted from the results of prolonging them in pairs. This fact provides the third check on the applicability of the critical path method to a given set of data.

Prolonging several processes which are on a path together can provide information about the order in which the processes are executed. The following theorem shows that if $X, Y$, and $Z$ are on a path in that order, that is, if $Y$ is between $X$ and $Z$, and if the prolongations $\Delta X, \Delta Y$, and $\Delta Z$ are not too small, then

$$
\Delta R T(\Delta X, \Delta Y, \Delta Z)=\Delta R T(\Delta X)+K(X Y)+\Delta R T(\Delta Y)+K(X Z)+\Delta R T(\Delta Z)
$$

Note that the term $K(X Z)$ does not occur in the above expression, so we can learn that $Y$ is between $X$ and $Z$ in the following way. By prolonging one process at a time we can calculate $\Delta R T(\Delta X), \Delta R T(\Delta Y)$, and $\Delta R T(\Delta Z)$. Then by prolonging two of the processes at a time we can calculate $K(X Y), K(Y Z)$, and $K(X Z)$ from Eq. (22). Finally, we prolong all three processes together and try to fit the above equation with exactly two of the parameters $K(X Y), K(Y Z), K(X Z)$. The parameter left out corresponds to the first and last of the three processes.

If $X_{i}$ is a process we denote its starting point by $x_{i, 1}$ and its terminating point by $x_{i, 2}$. To avoid the use of double subscripts we will sometimes write $S(X Y)$ for $S_{X Y}$.

Theorem 5. Suppose $X_{1}<X_{2}<\cdots<X_{n}$ and suppose for every $i, i=1, \ldots, n$, 
$\Delta X_{i}$ is a prolongation of $X_{i}$ such that $\Delta X_{i} \geqslant S\left(X_{i} t\right)$. If when every process $X_{i}$ is prolonged by $\Delta X_{i}$ there is a critical path containing all of $X_{1}, \ldots, X_{n}$ then

$$
\Delta R T\left(\Delta X_{1}, \ldots, \Delta X_{n}\right)=\sum_{i=1}^{n} \Delta R T\left(\Delta X_{i}\right)+\sum_{i=1}^{n-1} K\left(X_{i} X_{i+1}\right) .
$$

The proof is a straightforward generalization of the proof for Theorem 2(b).

The following theorem considers the effect on reaction time of prolonging three processes at a time. The theorem considers all four possible arrangements of three processes in a graph, except for variations produced by relabeling (see Fig. 6). Note that the four possibilities can be distinguished from one another by prolonging the processes in pairs and using Theorem 2 . There is only one case for which we cannot predict the quantitative effect on reaction time of prolonging three processes from the effect of prolonging them singly and in pairs. This is when the three processes are on a path together and the prolongations are small, case (d) below. Of course, if the prolongations are large, Theorem 5 applies to this case.

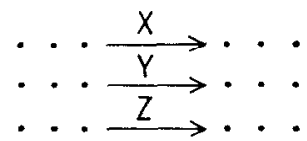

(a)

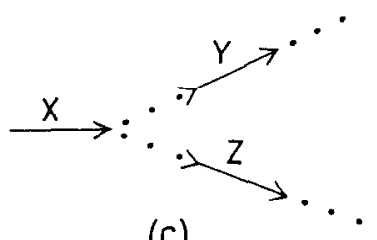

(c)

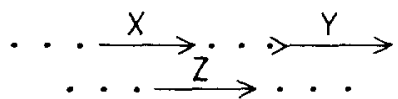

(b)

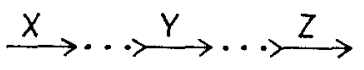

(d)

Fig. 6. The four possible arrangements of three processes in a graph.

Theorem 6. Let $X, Y$, and $Z$ be three processes.

(a) If no two of $X, Y$ and $Z$ are joined by a path (Fig. 6a)

$$
\Delta R T(\Delta X, \Delta Y, \Delta Z)=\max \{\Delta R T(\Delta X), \Delta R T(\Delta Y), \Delta R T(\Delta Z)\}
$$

(b) If $X$ precedes $Y$ on a path but no path joins $Z$ with $X$ or $Y$ (Fig. 6b),

$$
\Delta R T(\Delta X, \Delta Y, \Delta Z)=\max \{\Delta R T(\Delta X, \Delta Y), \Delta R T(\Delta Z)\} .
$$

(c) If $X$ precedes $Y$ on a path and $X$ precedes $Z$ on a path, but no path joins $Y$ and $Z$ (Fig. 6c),

$$
\Delta R T(\Delta X, \Delta Y, \Delta Z)=\max \{\Delta R T(\Delta X, \Delta Y), \Delta R T(\Delta X, \Delta Z)\}
$$


(d) If $X$ precedes $Y$ which precedes $Z$ on some path (Fig. 6d),

$$
\begin{aligned}
& \Delta R T(\Delta X, \Delta Y, \Delta Z) \\
& \quad=\max \left\{\Delta R T(\Delta X, \Delta Z),\left[\Delta Y-S_{Y t}+\left[\Delta Z-S_{Z Y}\right]^{+}+\left[\Delta X-S_{Y X}\right]^{+}\right]^{+}\right\} .
\end{aligned}
$$

Proof. Let $X, Y$, and $Z$ be arranged as indicated in each case.

(a) Using Theorem 2(a) to calculate the effect on $R T$ of prolonging $Y$ and $Z$ by $\Delta Y$ and $\Delta Z$, respectively,

$$
R T(\Delta X, \Delta Y, \Delta Z)=R T(\Delta X)+\max \left\{\left[\Delta Y-S_{Y t}(\Delta X)\right]^{+},\left[\Delta Z-S_{Z t}(\Delta X)\right]^{+}\right\} .
$$

Now using Eq. (20) to obtain $S_{Y t}(\Delta X)$ and $S_{Z t}(\Delta X)$ and using Eq. (14),

$$
\begin{aligned}
\Delta R T(\Delta X, \Delta Y, \Delta Z)= & {\left[\Delta X-S_{X t}\right]^{+}+\max \left\{\left[\Delta Y-S_{Y t}-\left[\Delta X-S_{X t}\right]^{+}\right]^{+}\right.} \\
& {\left.\left[\Delta Z-S_{Z t}-\left[\Delta X-S_{X t}\right]^{+}\right]^{+}\right\} . } \\
= & \max \{\Delta R T(\Delta X), \Delta R T(\Delta Y), \Delta R T(\Delta Z)\}
\end{aligned}
$$

where the last line follows from identity (13).

(b) Using Eq. (26) to calculate the effect on $R T$ of prolonging $X$ and $Y$ by $\Delta X$ and $\Delta Y$, respectively,

$R T(\Delta X, \Delta Y, \Delta Z)$

$$
=R T(\Delta Z)+\max \left\{\left[\Delta X-S_{X t}(\Delta Z)\right]^{+},\left[\Delta Y-S_{Y t}(\Delta Z)+\left[\Delta X-S_{X Y}(\Delta Z)\right]^{+}\right]^{+}\right\} .
$$

Using Eq. (20) and noting that $S_{X Y}(\Delta Z)=S_{X Y}$,

$$
\begin{aligned}
\Delta R T(\Delta X, \Delta Y, \Delta Z)= & {\left[\Delta Z-S_{Z t}\right]^{+}+\max \left\{\left[\Delta X-S_{X t}-\left[\Delta Z-S_{Z t}\right]^{+}\right]^{+},\right.} \\
& {\left.\left[\Delta Y-S_{Y t}-\left[\Delta Z-S_{Z t}\right]^{+}+\left[\Delta X-S_{X Y}\right]^{+}\right]^{+}\right\} } \\
= & \max \{\Delta R T(\Delta Z), \Delta R T(\Delta X, \Delta Y)\},
\end{aligned}
$$

where the last line follows from identity (13) and Eq. (26).

(c) Using Theorem 2(a) to calculate the effect on $R T$ of prolonging $Y$ and $Z$ by $\Delta Y$ and $\Delta Z$, respectively,

$$
R T(\Delta X, \Delta Y, \Delta Z)=R T(\Delta X)+\max \left\{\left[\Delta Y-S_{Y t}(\Delta X)\right]^{+},\left[\Delta Z-S_{Z t}(\Delta X)\right]^{+}\right\} .
$$

Using Eq. (24) and identity (13),

$$
\begin{aligned}
& \Delta R T(\Delta X, \Delta Y, \Delta Z) \\
&= {\left[\Delta X-S_{X t}\right]^{+}+\max \left\{\left[\Delta Y-S_{Y t}-\left[\Delta X-S_{X t}\right]^{+}+\left[\Delta X-S_{X Y}\right]^{+}\right]^{+},\right.} \\
& {\left.\left[\Delta Z-S_{Z t}-\left[\Delta X-S_{X t}\right]^{+}+\left[\Delta X-S_{X Z}\right]^{+}\right]^{+}\right\} } \\
&= \max \{\Delta R T(\Delta X, \Delta Y), \Delta R T(\Delta X, \Delta Z)\} .
\end{aligned}
$$


(d) Using Eq. (25) to calculate the effect on $R T$ of prolonging $X$ and $Y$ by $\Delta X$ and $\Delta Y$, respectively,

$$
\begin{aligned}
R T(\Delta X, \Delta Y, \Delta Z)= & R T(\Delta Z)+\left[\Delta X-S_{X t}(\Delta Z)\right]^{+} \\
& +\left[\Delta Y-S_{Y t}(\Delta Z)-\left[\Delta X-S_{X t}(\Delta Z)\right]^{+}+\left[\Delta X-S_{X Y}(\Delta Z)\right]^{+}\right]^{+} .
\end{aligned}
$$

We apply Eq. (24) to the converse, noting that $S_{X Y}(\Delta Z)=S_{X Y}$, so

$$
\begin{aligned}
& \Delta R T(\Delta X, \Delta Y, \Delta Z) \\
&=\left[\Delta Z-S_{Z t}\right]^{+}+\left[\Delta X-S_{X t}-\left[\Delta Z-S_{Z t}\right]^{+}+\left[\Delta Z-S_{Z X}\right]^{+}\right]^{+} \\
&+\left[\Delta Y-S_{Y t}-\left[\Delta Z-S_{Z t}\right]^{+}+\left[\Delta Z-S_{Z Y}\right]^{+}\right. \\
&\left.-\left[\Delta X-S_{X t}-\left[\Delta Z-S_{Z t}\right]^{+}+\left[\Delta Z-S_{Z X}\right]^{+}\right]^{+}+\left[\Delta X-S_{X Y}\right]^{+}\right]^{+} \\
&= \max \left\{\Delta R T(\Delta X, \Delta Z),\left[\Delta Y-S_{Y t}+\left[\Delta Z-S_{Z Y}\right]^{+}+\left[\Delta X-S_{X Y}\right]^{+}\right]^{+}\right\},
\end{aligned}
$$

where the last line follows from identity (13).

Q.E.D.

Two other expressions which may be useful for case (d) are

(i) $\Delta R T(\Delta X, \Delta Y, \Delta Z)=\max \left\{\Delta R T(\Delta X, \Delta Y),\left[\Delta Z-S_{Z t}+\max \left\{\left[\Delta X-S_{X Z}\right]^{+}\right.\right.\right.$,

$$
\left.\left.\left.\left[\Delta Y-S_{Y Z}+\left[\Delta X-S_{X Y}\right]^{+}\right]^{+}\right\}\right]^{+}\right\} \text {. }
$$

(ii) $\Delta R T(\Delta X, \Delta Y, \Delta Z)=\max \left\{\Delta R T(\Delta Y, \Delta Z),\left[\Delta X-S_{X t}+\max \left\{\left[\Delta Z-S_{Z X}\right]^{+}\right.\right.\right.$,

$$
\left.\left.\left.\left[\Delta Y-S_{Y X}+\left[\Delta Z-S_{Z Y}\right]^{+}\right]^{+}\right\}\right]^{+}\right\} \text {. }
$$

\section{Processes in Serial and Parallel}

In both psychology and graph theory the terms serial and parallel are used in slightly different ways by different authors. For our definitions we say that $X$ and $Y$ are in series if they are on a path together and if a process other than $X$ or $Y$ can be on a path with $X$ if and only if it is on a path with $Y$. We have already defined processes $X$ and $Y$ to be in parallel if they start at the same point and terminate at the same point. The reader can easily show that $X$ and $Y$ are in parallel according to our definition if and only if they are not on a path together and if furthermore a process other than $X$ or $Y$ can be on a path with $X$ if and only if it is on a path with $Y$. Extensive work on the stochastic behavior of serial and parallel processes has been done by Townsend $(1971,1972,1976)$. In this section we show in Theorem 9 that processes $X$ and $Y$ are either in series or in parallel if and only if for every process $A$ other than $X$ or $Y, K(A X)=K(A Y)$. This theorem can be used to show that certain processes are not in series or in parallel. To establish that certain processes are in series or in parallel, however, would require knowing the coupled slack for every pair of processes.

In the proof of Theorem 9 we use the following proposition; we are presenting it separately here because it illustrates the fact that Eq. (23),

$$
K(X Y)=d(s t)-d\left(s y_{1}\right)-d\left(x_{2} t\right)+d\left(x_{2} y_{1}\right)
$$


generates a variety of statements about the network which can be tested by comparing the magnitudes of the coupled slacks with one another.

Theorem 7. Suppose $X$ precedes $Y$ which precedes $Z$.

(a) If $y_{2}$ is on the longest path from $x_{2}$ to $t$ then

$$
K(X Z)-K(Y Z) \geqslant 0 .
$$

(b) If $y_{2}$ is on the longest path from $x_{2}$ to $z_{1}$ then

$$
K(X Z)-K(Y Z) \leqslant 0 .
$$

Proof. From (23)

$$
K(X Z)-K(Y Z)=d\left(x_{2} z_{1}\right)-d\left(y_{2} z_{1}\right)-d\left(x_{2} t\right)+d\left(y_{2} t\right) .
$$

Adding and subtracting $d\left(x_{2} y_{2}\right)$ we find

$$
K(X Z)-K(Y Z)=\left\{d\left(x_{2} z_{1}\right)-d\left(x_{2} y_{2}\right)-d\left(y_{2} z_{1}\right)\right\}-\left\{d\left(x_{2} t\right)-d\left(x_{2} y_{2}\right)-d\left(y_{2} t\right)\right\}
$$

and the results follow immediately.

In the proof of Theorem 9 we use the following lemma, the proof of which is left to the reader.

Lemma 8. Suppose $u$ precedes $Q$ which precedes w. If $u, X_{1}, v_{1}, \ldots, X_{n}$, w is a path from $u$ to w and if for every $i, i=1, \ldots, n$ either $X_{i}=Q$ or $X_{i}$ is on a path with $Q$, then there is some $i$ such that $X_{i}=Q$.

Theorem 9. Two processes $X$ and $Y$ are in series or in parallel iff for every process $A$ other than $X$ or $Y, K(A X)=K(A Y)$.

Proof. Suppose $K(A X)=K(A Y)$ for every $A \neq X, Y$. Then $K(A X)=-\infty$ iff $K(A Y)=-\infty$, so $X$ and $Y$ must be either in serial or parallel.

For the proof in the other direction, suppose $X$ and $Y$ are in series; with no loss of generality we can assume $X$ precedes $Y$. Let $A$ be another process. We wish to prove that $K(A X)=K(A Y)$. By hypothesis $K(A X)=-\infty$ iff $K(A Y)=-\infty$, so if $A$ is not on a path with $X$ or $Y$ we are finished. Suppose $A$ is on a path with $X$ and $Y$. There are two cases :

(i) Suppose $X$ precedes $A$ which precedes $Y$. Any process on the longest path from $x_{2}$ to $t$ is on a path with $X$ and, by hypothesis, is either $Y$ itself or is on a path with $Y$. Then by the preceding lemma, $Y$ is on the longest path from $x_{2}$ to $t$. Likewise, $X$ is on the longest path from $s$ to $y_{1}$.

Therefore, from Eq. (8)

$$
S_{X t}=d(s t)-\left[d\left(s x_{1}\right)+d(X)+d\left(x_{2} y_{1}\right)+d(Y)+d\left(y_{2} t\right)\right]=S_{Y t} .
$$


Furthermore, $S_{X_{A}}=0$ because any process on the longest path from $s$ to $a_{1}$ is on a path with $Y$ and hence on a path with $X$. Then by the preceding lemma, $X$ is on the longest path from $s$ to $a_{1}$, so $S_{X_{A}}=0$. Likewise, $S_{Y A}^{\prime}=0$. Then

$$
K(X A)=S_{X t}-S_{X A}=S_{Y t}-S_{Y A}^{\prime}=K(Y A) .
$$

(ii) Suppose $A$ is not between $X$ and $Y$. Without loss of generality we can assume $A$ follows $Y$, which follows $X$. Since $X$ and $Y$ are in series, by the preceding lemma $Y$ is on the longest path from $x_{2}$ to $t$ and $Y$ is also on the longest path from $x_{2}$ to $a_{1}$. Then by Theorem 7, $K(X A)-K(Y A)=0$.

The proof for $X$ and $Y$ in parallel is left to the reader.

Q.E.D.

\section{Analysis of a Stroop Task}

If two stimuli are presented simultaneously, or in rapid succession, and a subject responds to one or to both, his responses usually take longer than they do when he responds to each stimulus presented separately (Kantowitz, 1974; Kerr, 1973). The phenomenon is called "psychological refractoriness" after Telford's (1931) hypothesis, now discarded, that after processing one stimulus there is a "psychological refractory period," like the refractory period for neurons, during which a second stimulus cannot be processed. A notable example of interference while processing two stimuli is the Stroop effect (Stroop, 1935). If a color name, say green, is written in colored ink, say red ink, the time required to name the ink color is inordinately long.

We use the critical path method to analyze a Stroop-like experiment by Greenwald (1972), in which he manipulated two factors known to affect refractoriness, the difficulty of the decisions in the task and the degree of conflict in the responses. Response conflict occurs if, for example, one response is to press up while the other is to say "down"; such conflict is usually considered to produce reaction time delays by affecting activities concerned with responding (Kantowitz, 1974; Keele, 1973). Decisions have a role in refractoriness according to Welford's $(1952,1967)$ single channel theory because each stimulus must use a limited capacity decision mechanism and the mechanism can process only one stimulus at a time. Even if two stimuli are perceived simultaneously, each must wait its turn to use the central decision mechanism, and the waiting causes a delay in responding.

In Greenwald's experiment, subjects were presented with two stimuli almost simultaneously and responded to each. On each trial, the word "left" or the word "right" was presented through earphones and within a few milliseconds of the word's onset an arrow pointing either to the left or to the right was presented on a television monitor. Responses were made by speaking the word "left" or "right" and by moving the toggle switch to the left or right. In the high ideomotor compatibility condition, subjects moved the toggle switch in the direction indicated by the arrow and repeated the stimulus word. In the low ideomotor compatibility condition, the responses were interchanged, that is, subjects named the direction indicated by the arrow and moved the toggle switch in the direction 
indicated by the word. (Subjects never had to make a right response to a stimulus indicating left, or vice versa.) A different group of eight subjccts was run in each compatibility condition.

Reaction times were measured from the onset of the stimuli to the onset of each response. We let $R T_{\mathrm{v}}$ and $R T_{\mathrm{v}}^{*}$ be the reaction times for the verbal responses in the low and high ideomotor compatibility conditions, respectively; $R T_{\mathrm{m}}$ and $R T_{\mathrm{m}}^{*}$ are defined similarly for the manual responses. Subjects were not instructed to respond in any order, but the verbal response was usually produced after the manual response.

Our analysis is based on the reaction times for correct responses gathered on the last day of the experiment. ${ }^{1}$ The data from the last day, when subjects were highly practiced,

TABLE 2

Reaction Times and Differences in Reaction Times in Greenwald's Experiment ${ }^{a}$

\begin{tabular}{|c|c|c|c|c|}
\hline \multicolumn{4}{|c|}{ Low ideomotor compatibility } & \multirow[b]{2}{*}{$\Delta R T_{\mathrm{v}}$} \\
\hline Condition & $R T_{\mathrm{m}}$ & $R T_{\mathrm{v}}$ & $\Delta R T_{\mathrm{m}}$ & \\
\hline$\Delta D_{\mathrm{W}}, \Delta D_{\mathrm{A}}, \Delta C$ & 574 & 703 & 395 & 487 \\
\hline$\Delta D_{\mathrm{W}}, \Delta D_{\mathrm{A}}$ & 465 & 579 & 286 & 363 \\
\hline$\Delta D_{\mathrm{W}}, \Delta C$ & 358 & 465 & 179 & 249 \\
\hline$\Delta D_{\mathrm{w}}$ & 325 & 411 & 146 & 195 \\
\hline$\Delta D_{\mathrm{A}}, \Delta C$ & 309 & 424 & 130 & 208 \\
\hline$\Delta D_{\mathrm{A}}$ & 292 & 411 & 113 & 195 \\
\hline$\Delta C$ & 182 & 252 & 3 & 36 \\
\hline Baseline $R T$ & 179 & 216 & - & - \\
\hline \multicolumn{5}{|c|}{ High ideomotor compatibility } \\
\hline Condition & $R T_{\mathrm{m}}^{*}$ & $R T_{\vee}^{*}$ & $\Delta R T_{\mathrm{m}}^{*}$ & $\Delta R T_{*}^{*}$ \\
\hline$\Delta D_{\mathrm{W}}, \Delta D_{\mathrm{A}}, \Delta C$ & 297 & 351 & 124 & 126 \\
\hline$\Delta D_{\mathrm{w}}, \Delta D_{\mathrm{A}}$ & 295 & 343 & 122 & 118 \\
\hline$\Delta D_{\mathrm{w}}, \Delta C$ & 293 & 341 & 120 & 116 \\
\hline$\Delta D_{\mathrm{w}}$ & 292 & 331 & 119 & 106 \\
\hline$\Delta D_{\mathrm{A}}, \Delta C$ & 245 & 317 & 72 & 92 \\
\hline$\Delta D_{\mathrm{A}}$ & 231 & 293 & 58 & 68 \\
\hline$\Delta C$ & 188 & 246 & 15 & 21 \\
\hline Baseline $R T$ & 173 & 225 & 一 & - \\
\hline
\end{tabular}

a Times are in milliseconds. The $\Delta R T$ columns are formed from the corresponding $R T$ columns by subtracting the baseline $R T$ from each entry.

${ }^{1}$ Greenwald, A. G. Personal communication, April 14, 1977. 
give a slightly better fit to the equations and reveal more of the structure of the networks than Grecnwald's (1972) published data which were averaged over all 4 days.

The reaction times and changes in reaction times in the various conditions of the experiment are given in Table 2 . All measurements are in milliseconds.

In some blocks of trials, each stimulus could indicate left or right with equal probability, independently of the other; in other blocks, one stimulus was always the same, while the other varied randomly between left and right; finally, in some blocks, both stimuli were always the same. In a condition if the word or the arrow required a choice between two alternatives there is a $\Delta D_{\mathrm{W}}$ or a $\Delta D_{\mathrm{A}}$, respectively, in the corresponding row of Table 2.

On some trials within a block, the arrow and the word indicated different directions, so there was response conflict, and on other trials, the stimuli agreed, so there was no response conflict. Let $\Delta C$ denote the presence of conflict.

In our analysis of Greenwald's experiment, we consider the processes affected by the experimental manipulations in pairs and try to determine whether the processes in a pair are on a path or not. For processes $X$ and $Y$ prolonged by $\Delta X$ and $\Delta Y$, respectivcly, we see which of the following equations holds:

(a) $\Delta R T(\Delta X, \Delta Y)-\max \left\{\Delta R T(\Delta X), \Delta R T(\Delta Y)_{\}}\right.$,

(b) $\Delta R T(\Delta X, \Delta Y)=\Delta R T(\Delta X)+\Delta R T(\Delta Y)+K(X Y)$.

We use $K_{\mathrm{v}}(X Y)$ and $K_{\mathrm{m}}(X Y)$ in Eq. (22) to denote the coupled slack with respect to the verbal and the manual responses, respectively. The reader recalls from Theorem 2 that Eq. (21) holds only if $X$ and $Y$ are not joined by a path or if $X$ and $Y$, having negative coupled slack, are joined by a path in a Wheatstone bridge arrangement(Fig. 5, Theorem 3) and the prolongations $\Delta X$ and $\Delta Y$ are relatively small. Equation (22) holds if and only if $X$ and $Y$ are on a path together and $\Delta X$ and $\Delta Y$ are relatively large. Occasionally we go into more detail using Eq. (26).

To keep our discussion simple, we do not consider questions of parameter estimation and simply substitute data into equations in a straightforward way. More sophisticated techniques would improve our estimates and would, of course, change the amount of etror we report, although not substantially.

\section{Effects of the Number of Alternatives}

Let the process affected by the number of possible responses to the arrow be $D_{\mathrm{A}}$; presumably $D_{\mathrm{A}}$ is the process of deciding which response the arrow requires. Likewise, let $D_{\mathrm{W}}$ be the process affected by the number of possible responses to the word. Table 2 gives the effects on reaction times of prolonging $D_{\mathrm{w}}$ and $D_{\mathrm{A}}$ alone and in combination.

Low ideomotor compatibility. Let us begin with the low ideomotor compatibility condition and consider $R T_{\mathrm{v}}$, the reaction time for the verbal response to the arrow. We see from Table 2 that Eq. (22) holds for $\Delta R T_{\mathrm{v}}\left(\Delta D_{\mathrm{W}}, \Delta D_{\mathrm{A}}\right)$ with $K_{\mathrm{v}}\left(D_{\mathrm{W}} D_{\mathrm{A}}\right)=-27$; i.e., $363=195+195-27$. We conclude that $D_{\mathrm{W}}$ and $D_{\mathrm{A}}$ are on a path together, one preceding the other in accordance with single channel theory. If $D_{\mathrm{A}}$ and $D_{\mathrm{W}}$ were not 
on a path together, Eq. (2I) would hold, but (21) is in error by 168 . Since $K_{\mathrm{v}}\left(D_{\mathrm{w}} D_{\mathrm{A}}\right)$ is negative, $D_{\mathrm{w}}$ and $D_{\mathrm{A}}$ are arranged in a Wheatstone bridge like the network illustrated in Fig. 7, or its converse.

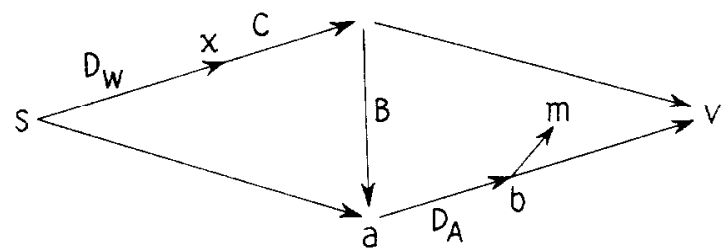

FIG. 7. A network for the low ideomoter compatibility condition. Only processes required to explain Greenwald's data are included. The data do not indicate the ordering of $C$ and $D_{A}$. The longest path from $s$ to $m$, not illustrated here, does not contain $C, D_{H^{-}}$, or $D_{A}$.

While $K_{\mathrm{v}}\left(D_{\mathrm{w}} D_{\mathrm{A}}\right)$ is not much less than zero, our inference that $D_{\mathrm{W}}$ and $D_{\mathrm{A}}$ are arranged in a Wheatstone bridge is consistent with the results we obtain in the high ideomotor compatibility condition.

For the manual response in the low ideomotor compatibility condition, Eq. (22) holds for $\Delta R T_{\mathrm{m}}\left(\Delta D_{\mathrm{w}}, \Delta D_{\mathrm{A}}\right)$ with $K_{\mathrm{m}}\left(D_{\mathrm{w}} D_{\mathrm{A}}\right)=27$, i.e., $286=146+113+27$. (The fact that $K_{\mathrm{v}}\left(D_{\mathrm{w}} D_{\mathrm{A}}\right)$ was negative does not imply that $K_{\mathrm{m}}\left(D_{\mathrm{w}} D_{\mathrm{A}}\right)$ must be negative.) The idea that $D_{\mathrm{W}}$ and $D_{\mathrm{A}}$ are on a path together in the low ideomotor compatibility condition is strongly supported; Eq. (21), which would hold otherwise, is in error by 140 .

Which comes first, $D_{\mathrm{w}}$ or $D_{\mathrm{A}}$ ? By Eq. (29), if $D_{\mathrm{A}}$ precedes $D_{\mathrm{w}}$ we would expect

$$
\Delta R T_{\mathrm{v}}\left(\Delta D_{\mathrm{w}}, \Delta D_{\mathrm{A}}\right)-\Delta R T_{\mathrm{v}}\left(\Delta D_{\mathrm{w}}\right)=\Delta R T_{\mathrm{m}}\left(\Delta D_{\mathrm{w}}, \Delta D_{\mathrm{A}}\right)-\Delta R T_{\mathrm{m}}\left(\Delta D_{\mathrm{w}}\right)
$$

Calculating from Table 2 we find that the left-hand side is 168 while the right-hand side is 140 , so this equation does not hold very well. On the other hand, if $D_{\mathrm{w}}$ precedes $D_{\mathrm{A}}$ we would expect

$$
\Delta R T_{\mathrm{v}}\left(\Delta D_{\mathrm{W}}, \Delta D_{\mathrm{A}}\right)-\Delta R T_{\mathrm{v}}\left(\Delta D_{\mathrm{A}}\right)=\Delta R T_{\mathrm{m}}\left(\Delta D_{\mathrm{w}}, \Delta D_{\mathrm{A}}\right)-\Delta R T_{\mathrm{m}}\left(\Delta D_{\mathrm{A}}\right)
$$

The left-hand side is 168 and the right-hiand side is 173 ; since the error is only 5 we conclude that $D_{\mathrm{W}}$ precedes $D_{\mathrm{A}}$.

Let us investigate the location of the onset of the manual response with respect to $D_{\mathrm{W}}$ and $D_{\mathrm{A}}$. Let $b$ and $x$ be the terminal points of $D_{\mathrm{A}}$ and $D_{\mathrm{W}}$, respectively, and let $m$ and $v$ be the onsets of the manual and verbal responses, respectively (see Fig. 7). While we cannot locate point $m$ precisely, the following remarks restrict the possibilities: (a) Point $m$ is not on the longest path from $x$ to $v$, otherwise by Eq. (8) we would have $S_{x v}-S_{x m}=S_{m v}$, which must be nonnegative. But $S_{x v}-S_{x m}=\Delta R T_{m}\left(\Delta D_{\mathrm{w}}\right)-$ $\Delta R T_{\mathrm{v}}\left(\Delta D_{\mathrm{w}}\right)=-49$ which is negative; (b) likewisc, $m$ is not on the longest path from $b$ to $v$; (c) process $D_{\mathrm{A}}$ precedes both $m$ and $v$, otherwise prolonging $D_{\mathrm{A}}$ would not increase both the manual and the verbal reaction times; and (d) remarks (b) and (c) indicate that 
the longest path from $b$ to $m$ must branch from the longest path from $b$ to $v$ at some point; we have placed the branching point at $b$ in Fig. 7, but the point may occur after $b$.

High ideomotor compatibility. Turning to the high ideomotor compatibility condition we see that $\Delta R T_{\mathrm{r}}^{*}\left(\Delta D_{\mathrm{w}}, \Delta D_{\mathrm{A}}\right)$ is about equal to the maximum of $\Delta R T_{\mathrm{r}}^{*}\left(\Delta D_{\mathrm{w}}\right)$ and $\Delta R T_{\mathrm{r}}^{*}\left(\Delta D_{\mathrm{A}}\right)$, i.e., $118=\max \{106,68\}$ with an error of 12 . Since Eq. (21) holds, it may be that $D_{\mathrm{w}}$ and $D_{\mathrm{A}}$ are, as in Fig. 8, not joined by a path or it may be that $D_{\mathrm{w}}$ and $D_{\mathrm{A}}$ have negative coupled slack and are arranged in a Wheatstone bridge like the network in Fig. 7 or its converse. Whether or not $D_{\mathrm{W}}$ and $D_{\mathrm{A}}$ are on a path together, each precedes $v$, the onset of the verbal response, since prolonging either of them individually prolongs $R T_{\mathrm{v}}^{*}$.

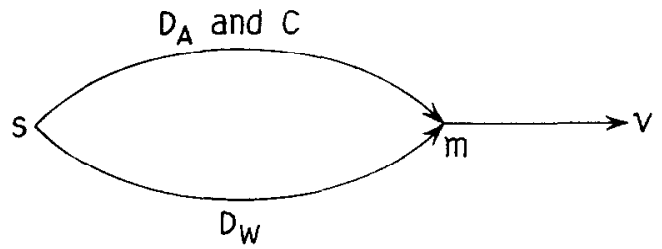

FIG. 8. A network for the high ideomotor compatibility condition. The order of $C$ and $D_{A}$ is not indicated by the data.

Considering the manual response, we find that Eq. (21) holds for $R T_{\mathrm{m}}^{*}\left(\Delta D_{\mathrm{W}}, \Delta D_{\mathrm{A}}\right)$, i.e., $122=\max \{119,58\}$ with an error of 3 . Furthermore, $D_{\mathrm{w}}$ and $D_{\mathrm{A}}$ both precede $m$, the onset of the manual response, since prolonging either $D_{\mathrm{W}}$ or $D_{\mathrm{A}}$ individually prolongs $R T_{\mathrm{m}}^{*}$. A network including $D_{\mathrm{w}}, D_{\mathrm{A}}$ and both responses in the high ideomotor compatibility condition is shown in Fig. 8. The actual task network may, of course, contain processes not indicated in the figure, in particular, there are almost certainly perceptual processes coming before the decisions.

Networks other than the one in Fig 8 are possible Recall that $x$ and $b$ are the terminal points of $D_{\mathrm{W}}$ and $D_{\mathrm{A}}$, respectively. The point $m$ could be on the longest path from $b$ to $v$, on the longest path from $x$ to $v$, or on both. Furthermore, $D_{\mathrm{A}}$ may precede $D_{\mathrm{w}}$ or $D_{\mathrm{A}}$ might not be on a path with $D_{\mathrm{w}}$. No strong conclusions about these possibilities can be drawn from the data.

Although it is possible that the networks in the two conditions may have the same shape, the locations of some of their processes must be different, since in one condition the verbal response is made to the word, while in the other is made to the arrow.

Process $B$ in Fig. 7, which may have zero duration, indicates that one decision precedes the other. According to Welford's single channel theory the bridge $B$ would be present in the high ideomotor compatibility condition, although the prolongations $\Delta D_{\mathrm{W}}$ and $\Delta D_{\mathrm{A}}$ may not have been long enough to reveal its existence. According to Greenwald's theory, on the other hand, in the high ideomotor compatibility condition the feedback from the response resembles the stimulus, and this unusual characteristic of the condition allows the limited capacity decision mechanism postulated by Welford to be bypassed. Further experiments are required to establish the existence or nonexistence of a path between decisions when there is high ideomotor compatibility. 


\section{Effects of Response Conflict}

Low ideomotor compatibility. Let $C$ be the process affected by response conflict. In the low ideomotor compatibility condition we find that $C$ is on a path with $D_{\mathrm{w}}$ since Eq. (22) holds for $\Delta R T_{\mathrm{v}}\left(\Delta C, \Delta D_{\mathrm{w}}\right)$ with $K_{\mathrm{v}}\left(C D_{\mathrm{w}}\right)=18$, i.e., $249=195+36+18$. Note that $C$ precedes the manual response because $R T_{\mathrm{m}}\left(\Delta D_{\mathrm{w}}, \Delta C\right)>R T_{\mathrm{m}}\left(\Delta D_{\mathrm{w}}\right)$, that is, the manual reaction time sometimes changes when $C$ is prolonged. Since $\Delta R T_{\mathrm{m}}(\Delta C)$ equals 3 , it is barely greater than zero so the hypotheses of Theorem 2 are not satisfied. Therefore Eq. (22) cannot be used to find the value of $K_{\mathrm{m}}\left(C D_{\mathrm{w}}\right)$. We show shortly that $K_{m}\left(C D_{\mathrm{w}}\right)=67$, but first we show that $D_{\mathrm{w}}$ precedes $C$.

The data show that $\Delta R T_{\mathrm{m}}\left(\Delta C, \Delta D_{\mathrm{w}}\right)>\Delta R T_{\mathrm{m}}\left(\Delta D_{\mathrm{w}}\right)>0$ and $\Delta R T_{\mathrm{m}}(\Delta C)$ is close to zero. Looking for this combination in Table 1 we find that $K_{\mathrm{m}}\left(C D_{\mathrm{w}}\right)>0$. Furthermore, if $C$ precedes $D_{\mathrm{w}}$ (which we show is wrong) then line 8 of Table 1 shows that $\Delta D_{\mathrm{w}}>S_{\mathrm{m}}^{\prime}\left(D_{\mathrm{w}} C\right), S\left(D_{\mathrm{w}} m\right)$. Table 1 , line 9 , shows also that for the verbal $R T, \Delta D_{\mathrm{w}}>$ $S_{\mathrm{v}}^{\prime}\left(D_{\mathrm{w}} C\right), S\left(D_{\mathrm{w}} v\right)$. Then Theorem $4(\mathrm{~b})$ applies, and if $C$ precedes $D_{\mathrm{w}}$ we should have

$$
\Delta R T_{\mathrm{v}}\left(\Delta C, \Delta D_{\mathrm{w}}\right)-\Delta R T_{\mathrm{v}}\left(\Delta D_{\mathrm{w}}\right)=\Delta R T_{\mathrm{m}}\left(\Delta C, \Delta D_{\mathrm{w}}\right)-\Delta R T_{\mathrm{m}}\left(\Delta D_{\mathrm{w}}\right) .
$$

But the left-hand side is 54 while the right-hand side is 33 ; the error is 21 so the equation does not hold well. We conclude that the hypothesis that $C$ precedes $D_{\mathrm{w}}$ is wrong, hence $D_{\mathrm{w}}$ precedes $C$. We test this conclusion below.

Now, from Theorem 4(a),

$$
\Delta R T_{\mathrm{m}}\left(\Delta D_{\mathrm{W}}\right)+K_{\mathrm{m}}\left(D_{\mathrm{W}} C\right)=\Delta R T_{v}\left(\Delta D_{\mathrm{W}}\right)+K_{v}\left(D_{\mathrm{W}} C\right) .
$$

Calculating, we find that $K_{\mathrm{m}}\left(D_{\mathrm{w}} C\right)=67$.

Is $C$ on a path with $D_{\mathrm{A}}$ ? If so, then Eq. (31) should hold. Suppose $D_{\mathrm{A}}$ precedes $C$. Then by Eq. (31)

$$
K_{\mathrm{v}}\left(D_{\mathrm{w}} C\right)-K_{\mathrm{v}}\left(D_{\mathrm{w}} D_{\mathrm{A}}\right)=K_{\mathrm{m}}\left(D_{\mathrm{w}} C\right)-K_{\mathrm{m}}\left(D_{\mathrm{w}} D_{\mathrm{A}}\right) .
$$

The right-hand side is 45 and the left-hand side is 40 . This equation validates our conclusion that $D_{\mathrm{w}}$ precedes $C$ and that $K_{\mathrm{m}}\left(C D_{\mathrm{w}}\right)=67$. Furthermore, the equation shows that $C$ is indeed on a path with $D_{\mathrm{A}}$. We do not know whether $C$ precedes or follows $D_{\mathrm{A}}$, however, since the above equation is equivalent for both cases.

Which equation, (21) or (22), applies to $C$ and $D_{\mathrm{A}}$ ? For the verbal reaction times, Eq. (22) holds for $C$ and $D_{\mathrm{A}}$ with $K_{\mathrm{v}}\left(C D_{\mathrm{A}}\right)=-23$, i.e., $208=195+36-23$. However, one could argue that Eq. (21) holds instead, since $208=\max \{195,36\}$ with an error of 13 . Either way since $C$ and $D_{\mathrm{A}}$ are on a path together they are on a Wheatstone bridge.

Since $\Delta R T_{\mathrm{m}}(\Delta C)$ is nearly zero we cannot apply Eq. (22) to $\Delta R T_{\mathrm{m}}\left(\Delta C, \Delta D_{\mathrm{A}}\right)$. Equation (21) holds approximately, however, since $130=\max \{113,3\}$ with an error of 17 .

Incidentally, none of the terminating points of $C, D_{\mathrm{w}}$, or $D_{\mathrm{A}}$ is on the critical path from $s$ to $m$ because $K_{\mathrm{m}}\left(C D_{\mathrm{w}}\right)$ and $K_{\mathrm{m}}\left(D_{\mathrm{w}} D_{\mathrm{A}}\right)$ are both positive. Therefore each of these processes has positive total slack with respect to $m$. 
High ideomotor compatibility. In this section we establish an important fact: The scheduling of mental processes is not fixed but depends on the experimental task. In particular, the positions of $D_{\mathrm{W}}$ and $D_{\mathrm{A}}$ are probably interchanged in the high and low ideomotor compatibility conditions.

Recall that in the low ideomotor compatibility condition $C$ and $D_{\mathrm{W}}$ are on a path together but not in a Wheatstone bridge, while $C$ and $D_{\mathrm{A}}$ are in a Wheatstone bridge. In the high ideomotor compatibility condition, it is $C$ and $D_{\mathrm{A}}$ which are on a path together, but not in a Wheatstone bridge, while $C$ and $D_{\mathrm{W}}$ are either in a Wheatstone bridge or not on a path together.

Here are the details. Equation (21) holds for $C$ and $D_{\mathrm{W}}$. From Table 2, $\Delta R T_{\mathrm{v}}^{*}\left(\Delta D_{\mathrm{w}}, \Delta C\right)$ is about equal to the maximum of $\Delta R T_{\mathrm{v}}^{*}\left(\Delta D_{\mathrm{w}}\right)$ and $\Delta R T_{\mathrm{v}}^{*}(\Delta C)$, i.e., $116=\max \{106,21\}$ with an error of 10 . And $\Delta R T_{\mathrm{m}}^{*}\left(\Delta D_{\mathrm{w}}, \Delta C\right)$ is about equal to the maximum of $\Delta R T_{\mathrm{m}}^{*}\left(\Delta D_{\mathrm{w}}\right)$ and $\Delta R T_{\mathrm{m}}^{*}(\Delta C)$, i.e., $120=\max \{119,15\}$ with an error of 1 . We conclude that $C$ and $D_{\mathrm{w}}$ are either not on a path together or are on a path together in a Wheatstone bridge.

Processes $C$ and $D_{\mathrm{A}}$ have additive effects in the high ideomotor compatibility condition. Equation (22) holds for the verbal reaction times; $\Delta R T_{\nabla}^{*}\left(\Delta C, \Delta D_{\mathrm{A}}\right)$ is about equal to the sum of $\Delta R T_{\mathrm{v}}^{*}(\Delta C)$ and $\Delta R T_{\mathrm{v}}^{*}\left(\Delta D_{\mathrm{A}}\right)$, i.e., $92=68+21+3$. Likewise, for the manual reaction times, $\Delta R T_{\mathrm{m}}^{*}\left(\Delta C, \Delta D_{\mathrm{A}}\right)$ is about equal to the sum of $\Delta R T_{\mathrm{m}}^{*}(\Delta C)$ and $\Delta R T_{\mathrm{m}}^{*}\left(\Delta D_{\mathrm{A}}\right)$, i.e., $72=58+15-1$. We conclude that $C$ and $D_{\mathrm{A}}$ are on a path together.

We do not have enough information to order $C$ and $D_{\mathrm{A}}$. Theorem 4 holds almost as well for $C$ preceding $D_{\mathrm{A}}$ as for $D_{\mathrm{A}}$ preceding $C$, and it is hard to make a strong case for one order over the other.

Figure 8 illustrates the scheduling of the processes in the high ideomotor compatibility condition. The ordering of $C$ and $D_{\mathrm{A}}$ has been left unspecified. For simplicity we have drawn $m$ on the longest path from the terminating points of $D_{\mathrm{W}}, D_{\mathrm{A}}$, and $C$; nothing in the data rules out this possibility.

\section{Discussion}

The interchange of the roles of $D_{\mathrm{W}}$ and $D_{\mathrm{A}}$ in the two compatibility conditions has important implications for theories of the Stroop effect. Many theories, e.g., Morton (1969), state that in the Stroop task the word automatically has access to the single channel before the color. Greenwald's data complicate the picture. We do not know if one decision precedes the other in the high ideomotor condition. But if one decision does come first it is probably the decision about the arrow. Note that since the stimuli are the same in the two ideomotor compatibility conditions, the positions of the decisions have been interchanged for reasons unrelated to stimulus features per se.

It may be a general rule that the subject makes his decisions in the order in which the corresponding responses occur. Greenwald's data are consistent with this rule. It is possible that the subjects learned to make their decisions in a certain order as the experiment went on. Part of the interference in the Stroop task as it is usually performed may occur because the subjects need to make two decisions at about the same time but do not have a system which automatically makes one decision first. Time may be required to decide what to decide first. 
We call $C$ the correlation process. It has properties very similar to those of a process hypothesized by Keele and Neill (in press) which coordinates information activated in memory. The coordinating process, like $C$, resolves response conflict. It is executed following processes concerned with the number of alternative stimuli. The coordinating process has, however, at least two functions which may not apply to our process $C$. First, in the view of Keele and Neill the coordinating process selects information from codes activated in memory. Second, the process coordinates information from the environment with information regarding goals. Process $C$ in the network model may be part of a more complicated system having the functions postulated by Keele and Neill.

\section{Durations of Paths}

Several equations provide information about the durations of the longest paths hetween points in the network. We present the highlights and leave the details to the interested reader. A thorough treatment of path durations requires a more sophisticated procedure for estimating parameters. We have chosen our estimates of coupled slack to make Eq. (22) hold exactly when it applies, but then other equations hold with some error. More sophisticated estimates would distribute the error more uniformly over the equations.

We have an equation of form (22) for every coupled slack $K(X Y)$, and these provide bounds on path durations. For example, in the low ideomotor compatibility condition $K_{v}\left(D_{\mathrm{W}} D_{\mathrm{A}}\right)=-27$. Recall that $a$ is the starting point of $D_{\mathrm{A}}$ and $x$ is the terminal point of $D_{\mathrm{W}}$. Then by (23)

$$
d(s v)-d(s a)-d(x v)+d(x a)=-27 .
$$

Then since $d(s v)-d(x v) \geqslant 0$ we obtain

$$
d(s a) \geqslant 27
$$

Considerable information is provided by Eq. (30). We find, for example, recalling that $b$ is the terminating point of $D_{\mathrm{A}}$,

$$
d(b v)-d(b m)=R T_{\mathrm{v}}\left(\Delta D_{\mathrm{A}}\right)-R T_{\mathrm{m}}\left(\Delta D_{\mathrm{A}}\right)=119 .
$$

Since $d(s v)=R T_{\mathrm{v}}=216$ while $d(b v) \geqslant 119$, over half of the verbal reaction time elapses after the subject has decided which verbal response is required.

The decision about the arrow is completed before 97 milliseconds elapse because

$$
\begin{aligned}
d(s b) & \leqslant d(s v)-d(b v) \\
& \leqslant 216-119=97
\end{aligned}
$$

We also find from Eq. (30) that

$$
d(x v)-d(x m)=R T_{\mathrm{v}}\left(\Delta D_{\mathrm{W}}\right)-R T_{\mathrm{m}}\left(\Delta D_{\mathrm{W}}\right)=86 .
$$


We cannot use Eq. (30) to learn about the durations of paths from $C$ to the responses because $\Delta R T_{\mathrm{m}}(\Delta C)$ is nearly zero.

\section{Prolonging Three Processes}

In the high ideomotor compatibility condition we know that $C$ and $D_{\mathrm{A}}$ are on a path together, but unfortunately we know little else. Suppose $D_{W}$ is not on a path with either $C$ or $D_{\mathrm{A}}$. Then Theorem 6(b) should apply. Since

$$
\Delta R T_{\mathrm{m}}^{*}\left(\Delta C, \Delta D_{\mathrm{w}}, \Delta D_{\mathrm{A}}\right)=\Delta R T_{\mathrm{m}}^{*}\left(\Delta D_{\mathrm{w}}\right)
$$

i.e., 124 is about equal to 119 with an error of 6 , the theorem holds. Theorem $6(\mathrm{~b})$ also holds tolerably well for the verbal reaction times since

$$
\Delta R T_{\mathrm{v}}^{*}\left(\Delta C, \Delta D_{\mathrm{W}}, \Delta D_{\mathrm{A}}\right)=\Delta R T_{\mathrm{v}}^{*}\left(\Delta D_{\mathrm{w}}\right)
$$

i.e., 126 is about equal to 106 with an error of 20 . The fact that the error is so large may indicate that $C, D_{\mathrm{W}}$ and $D_{\mathrm{A}}$ are all on a path together so that Theorem $6(\mathrm{~b})$ does not hold and Theorem 6(d) applies instead. The data are entirely consitent with Theorem 6(d).

The above equations provide support for the critical path method, but tell us little about the task network since several arrangements of $C, D_{\mathrm{w}}$, and $D_{\mathrm{A}}$ lead to equations of Theorem 6 which all hold reasonable well.

For the low ideomotor compatibility condition, we know $D_{\mathrm{W}}$ precedes $C$ and $D_{\mathrm{A}}$ and we know $C$ and $D_{\mathrm{A}}$ are on a path together. 'Therefore 'I'heorem $6(\mathrm{~d})$ should apply but we see that it does not hold with an acceptable error. The failure may occur because more practice is required, this particular case is the only case in which subjects must take two nontrivial decisions, the results of which conflict. This is only speculation, I do not know why the equation does not hold.

Consider Theorem 6(d). Suppose $D_{\mathrm{W}}$ precedes $C$ which precedes $D_{\mathrm{A}}$. (The fit is worse if we assume otherwise.) Theorem 6(d) states

$$
\begin{aligned}
\Delta R T_{\mathrm{m}}\left(\Delta D_{\mathrm{W}}, \Delta C, \Delta D_{\mathrm{A}}\right) \\
=\max \left\{\Delta R T\left(\Delta D_{\mathrm{W}}, \Delta C\right),\left[\Delta D_{\mathrm{A}}-S_{D_{\mathbf{A}} \mathrm{m}}+\max \left\{\left[\Delta D_{\mathrm{W}}-S_{D_{\mathbf{W}} D_{\mathbf{A}}}\right]^{+}\right.\right.\right. \\
\left.\left.\left.\quad\left[\Delta C-S_{C D_{\mathbf{A}}}+\left[\Delta D_{\mathrm{W}}-S_{D_{\mathbf{W}} C}\right]^{+}\right]^{+}\right\}\right]^{+}\right\} .
\end{aligned}
$$

Note that (a) $\Delta D_{\mathrm{W}}-S_{D_{\mathbf{W}_{\mathrm{A}} \mathrm{A}}}=\Delta R T_{\mathrm{m}}\left(\Delta D_{\mathrm{W}}\right)+K_{\mathrm{m}}\left(D_{\mathrm{W}} D_{\mathrm{A}}\right)=146+27=173$, (b) $\Delta D_{\mathrm{W}}-S_{\mathrm{D}_{\mathrm{W}} \mathrm{D}_{\mathrm{A}}}=\Delta R T_{\mathrm{m}}\left(\Delta D_{\mathrm{W}}^{\mathrm{A}}\right)+K_{\mathrm{m}}\left(D_{\mathrm{W}} C\right)=146+67=213$, and (c) if we assume $\Delta R T_{\mathrm{m}}\left(\Delta C, \Delta D_{\mathrm{A}}\right)>\Delta R T_{\mathrm{m}}\left(\Delta D_{\mathrm{A}}\right)$ then $\Delta C-S_{\mathrm{CD}_{\mathrm{A}}}=17$ from Table 1 . Substituting these values we find the left-hand side to be 395 while the right-hand side is $113 \div 17+$ $213=343$. The error is 52 which is not satisfactory. The equation is also in error for the verbal reaction times, the calculation is left to the reader. 


\section{ACKNOWLEDGMENTS}

I would like to thank Sylvan Kornblum and Henry Rueter for encouragement and many helpful discussions, Steven Bies and Marty Young for reading the manuscript, Anthony Greenwald for his generous assistance, and Jill Matuk, Dianne Nelson, and Dorene Root for skillful typing. This research was supported by a NIGMS Training Grant in Mathematical Psychology, GM-01231. I am indebted to one of the referees for simplifying the proof of Theorem 9.

\section{REFERENCES}

Christie, L. S., \& Luce, R. D. Decision structure and time relations in simple choice behavior. Bulletin of Mathematical Biophysics, 1956, 8, 89-112.

Donders, F. C. Die Schnelligkeit psychischer Processe. Archiv fur Anatomie und wissenschaftliche Medizin, 1868, 656-681.

Ford, L. R., \& Fulkerson, D. R. Flows in networks. Princeton, N.J.: Princeton University Press, 1962.

FulKerson, D. R. Expected critical path length in PERT networks. Operations Research, 1962, 10, 808-817.

Greenwald, A. G. On doing two things at once: Time sharing as a functional of ideomotor compatibility. Journal of Experimental Psychology, 1972, 94, 52-57.

Harary, F. Graph theory. Reading, Mass.: Addison-Wesley, 1969.

KaNTowitz, B. H. Double stimulation. In B. H. Kantowitz (F.d.), Human information processing: Tutorials in performance and cognition. Hillsdale, N.J.: Lawrence Erlbaum Assoc., 1974.

Keele, S. W. Attention and human performance. Pacific Palisades: Goodyear, 1973.

Kefle, S. W., \& Neill, W. T. Mechanisms of attention. In E. Carterette \& M. Friedman (Eds.), Handbook of perception. New York: Academic Press, in press. Vol. 9.

KelLeY, J. E., JR. Critical path planning and scheduling, mathematical basis. Operations Research, 1961, 9, 296-320.

KERR, B. Processing demands during mental operations. Memory and Cognition, 1973, 1, 401-412.

MrLxER, J. O. Effects of stimulus probability on encoding mechanisms in information processing tasks. Technical Report No. 56. Ann Arbor: The University of Michigan, Human Performance Center, June 1976.

Modor, J., \& Phillips, C. Project management with CPM and PERT. New York: Van Nostrand, 1970. 2nd ed.

Morton, J. Categories of interference: Verbal mediation and conflict in card sorting. British Journal of Psychology, 1969, 60, 329-346.

ShAPLEY, L. S. On network flow functions. Research Memorandum RM 1660 . The RAND corporation, March 16, 1959.

Siel.ken, R. L., Jr., Ringer, L. J., Hartley, H. O., \& Arseven, E. Statistical critical path analysis in acyclic stochastic networks: Statistical PERT. Project Themis Technical Report No. 48, Institute of Statistics, Texas A \& M University, November 1974.

STERnBERG, S. The discovery of processing stages: Extensions of Donder's method. In W. G. Koster (Ed.), Attention and performance II. Amsterdam: North-Holland, 1969a.

SteRnRfRG, S. Memory scanning: Mental processes revealed by reaction-time experiments. American Scientist, 1969b, 57, 421-457.

Stroop, J. R. Studies of interference in serial verbal reactions. Journal of Experimental Psychology, $1935,18,643-662$.

TELFORD, C. W. Refractory phase of voluntary and associative responses. Journal of Experimental Psychology, 1931, 14, 1-35.

Townsend, J. T. A note on the identifiability of parallel and serial processes. Perception and Psychophysics, 1971, 10, 161-163. 
Townsend, J. T. Some results concerning the identifiability of parallel and serial processes. British Journal of Mathematical and Statistical Psychology, 1972, 25, 168-199.

TowNSEND, J. T. Serial and within-stage independent parallel model equivalence on the minimum completion time. Journal of Mathematical Psychology, 1976, 14, 219-238.

Werst, J. D., \& Levy, F. K. A management guide to PERT/CPM. Englewood Cliffs, N.J.: PrenticeHall, 1969.

WELFORD, A. T. The "psychological refractory period" and the timing of high-speed performanceA review and a theory. British Journal of Psychology, 1952, 43, 2-19.

WElford, A. T. Single-channel operation in the brain. In A. F. Sanders (Ed.), Attention and performance I. Amsterdam: North-Holland, 1967.

ReCEIVED: May 16, 1977 